\title{
Energy budget for the larval development of Elminius modestus (Crustacea: Cirripedia)
}

\author{
J. Harms \\ TH Darmstadt, Zoologisches Institut; Schnittspahnstr. 10, \\ D-6100 Darmstadt, Federal Republic of Germany \\ and \\ Biologische Anstalt Helgoland (Meeresstation); \\ D-2192 Helgoland, Federal Republic of Germany
}

\begin{abstract}
Biomass (CHN), respiration rate and food uptake were estimated for the larval development of Elminius modestus at three temperatures $\left(12,18,24{ }^{\circ} \mathrm{C}\right)$. Mean values of dry weight, elemental composition and energy equivalents increased exponentially with the development from nauplius II to VI. Dry weight, elemental composition and energy content exhibited the highest values at $18^{\circ} \mathrm{C}$. Respiration rates increased with the larval stages expressed by a power function, but increased logarithmically with the dry weight of the larvae. The cypris larvae showed a reduced respiration rate compared with nauplius VI. The ingestion rate was measured at a concentration of 100 cells of Skeletonema costatum $\mu \mathrm{l}^{-1}$. At 12 and $18^{\circ} \mathrm{C}$ ingestion rates increased exponentially and at $24{ }^{\circ} \mathrm{C}$ by a logarithmic function. The fittings were used to estimate the energy budget of $E$. modestus during larval development. The energy content of the larvae increased during the development from nauplius II to $\mathrm{VI}$ by a factor of 21 at $12^{\circ} \mathrm{C}, 25$ at $24^{\circ} \mathrm{C}$ and 31 at $18^{\circ} \mathrm{C}$. The estimated energy content of the freshly metamorphosed barnacle is $100 \mathrm{~mJ}\left(12^{\circ} \mathrm{C}\right), 130 \mathrm{~mJ}\left(24^{\circ} \mathrm{C}\right)$ and $150 \mathrm{~mJ}\left(18^{\circ} \mathrm{C}\right)$. The assimilation- $(\mathrm{A} / \mathrm{I})$ and gross growth efficiencies $\left(\mathrm{K}_{1}\right)$ increased strongly during the development from nauplius II to VI (A/I: 6-14\% in nauplius II to $50-90 \%$ in nauplius VI; $\mathrm{K}_{1}: 4 \%$ in nauplius II to $75 \%$ in nauplius VI). The net growth efficiency $\left(\mathrm{K}_{2}\right)$ showed a relatively constant level ranging between 57 and $83 \%$.
\end{abstract}

\section{INTRODUCTION}

Elminius modestus was recently introduced to Europe (Bishop, 1947). Since then it has spread rapidly throughout this continent (see Harms, 1986). Larvae of E. modestus develop through six naupliar stages and finally metamorphose into the non-feeding cypris larva. The proposed function of pelagic larval stages is the dispersal to and the colonization of remote habitats, offering the advantage that a large number of geographically isolated and diverse habitats are reached where populations are at less risk with regard to density-independent mortalities caused by local catastrophes (Crisp. 1974,1976 ). Pelagic larvae suffer high mortality due to predation or lack of food. For this reason, the number of larvae produced are maximized, with the effect that the larvae contain only a small amount of energy when they hatch. The capacity of the cypris to metamorphose into juvenile barnacles depends entirely on the energy reserve accumulated by the naupliar stages (II-VI). So the cypris larva, which does not feed (Walley, 
1969), normally contains some $20 \%$ of its body weight as energy reserve (Holland \& Walker, 1975). Advances in the rearing technique of barnacles (Harms, 1984, 1985; Moyse, 1963; Tighe-Ford et al., 1970; Wisely, 1960) made sufficient numbers of larvae up to the cypris available for studying larval development. This study on the energy accumulation during the larval development of $E$. modestus describes and compares the $\mathrm{CHN}$-ratio, the oxygen and the food uptake of the single naupliar stages with data of other crustuaceans especially cirripedes.

\section{MATERIALS AND METHODS}

Rearing technique

The adults of Elminius modestus were collected in the intertidal zone of the island Helgoland (North Sea) and were kept in a flow-through system at a constant temperature of $12{ }^{\circ} \mathrm{C}$. Only freshly hatched larvae not older than $6 \mathrm{~h}$ were used for the experiments. Usually, the larvae had reached the second nauplius stage at this age. The first nauplius stage is not considered here, because it lasts only a few hours and does not feed (Barnes \& Barnes, 1958; Molenock \& Gomez, 1972; Moyse, 1963).

The larvae were reared in mass cultures (5-10-liter beakers at 12,18 and $24{ }^{\circ} \mathrm{C}$ with a concentration of 1 larva $\times \mathrm{ml}^{-1}$ in $0.45 \mu \mathrm{m}$ filtered seawater (31-33\% oS). Slight aeration provided equal distribution of the larvae and kept the algae in suspension. The larvae were fed with Skeletonema costatum $\left(100\right.$ cells $\left.\times \mu l^{-1}\right)$. Antibiotics were added at a concentration of $0.3 \mathrm{ml}$ Crystamycin $\times \mathrm{I}^{-1}$ seawater (Crystamycin $=300 \mathrm{mg}$ Penicillin $\mathrm{G}+500 \mathrm{mg}$ Streptomycin in $4 \mathrm{ml}$ destilled water). The culture medium was changed every second day. Before starting further experiments, the development of the larvae was checked and only simultaneously developing cultures were used.

\section{CHN-Analyses}

To determine dry weight and carbon-, nitrogen- and hydrogen-content, precounted numbers of larvae (Stage II: 1000 larvae; III: 600-800 larvae; IV: 500-600 larvae; V: 150 larvae; VI and cypris: 100 larvae) were pipetted onto pre-ashed and pre-weighed Whatman glass fibre filters. The seawater was vacuum-filtered; larvae were washed briefly with fresh water before being placed, on glass fibre filters, into silver cartridges. They were freeze dried at $<10^{-2} \mathrm{mbar}$ in a GT2 (Leybold Hereaus) for at least $12 \mathrm{~h}$. Dry weight was estimated on an electronic autobalance (Perkin-Elmer AD2). Carbon-, nitrogen- and hydrogen-content of larvae were determined by means of an Elemental Analyser Model 1106 (Carlo Erba Science). Energy equivalents were calculated using the formula given by Salonen et al. (1976) and were expressed in Joules.

\section{Respiration}

The respiration rate was measured at 12,18 and $24^{\circ} \mathrm{C}$ applying the Winkler method (Anger \& Jacobi, 1985; Dawirs, 1983; Grasshoff, 1976). Depending on their dry weight, 100 (Cypris and nauplius VI) to 1000 (nauplius II) larvae were confined in one Winkler bottle $\left(\approx 55 \mathrm{~cm}^{3}\right)$, containing $0.25 \mu \mathrm{m}$ filtered seawater $(31-33 \%$ oS). Each measurement 
included 10 experimental sets (with larvae) and 5 replicate blanks (without larvae) and ran for $24 \mathrm{~h}$. The decrease of oxygen concentration in the experiments was 5 to $20 \%$, which is believed to have no effect on larval respiration (Belman \& Childress, 1973; Dawirs, 1983) and can be measured accurately.

The oxygen uptake was calculated by the difference between the blanks and experimental sets. The oxygen consumption $\mathrm{mg} \mathrm{O} \mathrm{O}_{2} \times \mathrm{1}^{-1}$ can be converted into $\mathrm{ml}$ $\mathrm{O}_{2} \times 1^{-1}$ by the factor 0.7 (Crisp, 1984). The oxygen uptake was converted to energy loss by metabolic heat production (Gnaiger, 1983: $1 \mathrm{mg} \mathrm{O}_{2}=14.06$ Joule).

\section{Ingestion rate}

The algae concentration was measured using the haematocyte counting chamber of Neubauer (Guillard, 1978). A preliminary experiment had shown good agreement with the algae concentration measured by the Utermöhl method (Utermöhl, 1958) and the Neubauer chamber.

Each experimental set contained 300 larvae in $300 \mathrm{ml}$ filtered seawater $(0.25 \mu \mathrm{m})$ with a food concentration of 100 cells $\times \mu l^{-1}$ of Skeletonema costatum at the beginning of the experiments. No antibiotics were added. Each experiment included 10 replicates (with larvae) and 5 replicate blanks (without larvae). The algae concentration was measured after 12,18 and $24 \mathrm{~h}$ (at $12^{\circ} \mathrm{C}$ also after $36 \mathrm{~h}$ ). The ingestion rate was calculated by the method given by Frost (1972).

The ingested cells $\times$ day $^{-1}$ were used for the calculation of the energy uptake. Cells of $S$. costatum have a carbon content of $16.76 \pm 2.38 \mathrm{pg} \mathrm{C}$ (Hagmeier, personal communication). This value is comparable to the one given by Strathman (1967). One cell has therefore an energy content of $0.67 \mu \mathrm{J}$ (converted after Finlay \& Uhlig, 1981).

\section{Energy budget}

The energy budget for each nauplius stage and for the total larval development from nauplius II to cypris stage is given by the formula:

$$
\mathrm{I}=\mathrm{G}+\mathrm{Ex}+\mathrm{M}+\mathrm{E}_{\text {, }}
$$

where $G$ is the growth rate, Ex is the energy content of the exuviae and $M$ is the metabolism, which is estimated by the oxygen uptake. No experiments were performed on the excretion and egestion (E), thus this value is given by the difference of the assimilated energy (A) and the ingested food (I) (Dawirs, 1983): $\mathrm{E}=\mathrm{I}-\mathrm{A}$.

\section{Efficiencies}

Data on growth, food uptake and respiration, which have been estimated for the larvae of Elminius modestus, were used for the calculation of the following efficiencies:

Assimilation efficiency (A/1): calculated by

$$
\frac{\text { respiration rate }+ \text { growth rate }+ \text { exuviae }}{\text { ingestion rate }} \times 100
$$


it denotes the proportion of ingested food assimilated. Assimilation in this context has been defined as the sum of respiration and growth (Crisp, 1984).

Gross growth efficiency $\left(K_{1}\right)$ : calculated by

$$
\frac{\text { growth rate }+ \text { exuviae }}{\text { ingestion rate }} \times 100 \text {; }
$$

it denotes the proportion of ingested food converted to growth.

Net growth efficiency $\left(\mathrm{K}_{2 \mathrm{a}}\right)$ : calculated by

$$
\frac{\text { growth rate }+ \text { exuviae }}{\text { growth rate }+ \text { exuviae }+ \text { respiration rate }} \times 100 \text {; }
$$

or $\mathrm{K}_{2 \mathrm{~b}}$ :

$$
\frac{\text { growth rate }}{\text { growth rate }+ \text { exuviae + respiration rate }} \times 100 ;
$$

it denotes the proportion of assimilated food converted to growth.

The assimilation-, gross- and net growth efficiences $\left(A / I, K_{1}, K_{2 a}\right)$ were calculated with consideration of the exuviae, because the exuviae participate in the growth of the larvae. The calculation of the net growth efficiency $\mathrm{K}_{2 \mathrm{~b}}$ is based on the assumption that the energy content of the exuviae is wasted for the larvae.

\section{RESULTS}

\section{CHN-Analyses}

The mean values of dry weight (W), elemental composition $(\mathrm{C}, \mathrm{N}, \mathrm{H})$ and the energy equivalents $\left(\mathrm{mJ} \times \mathrm{Ind}^{-1}\right)$ (Table 1 ) of the naupliar stages increased exponentially during the development from nauplius II to VI (Table 2):

$$
\ln y=b+m t \text {, }
$$

$y=$ individual dry weight $(\mathrm{W})$, carbon $(\mathrm{C})$, nitrogen $(\mathrm{N})$, hydrogen $(\mathrm{H})$ or energy $(\mathrm{mJ} x$ Ind $\left.{ }^{-1}\right) ; t=$ time of development; $b$ and $m=$ constants. Dry weight (W) and elemental composition $\left(\mathrm{C}, \mathrm{N}, \mathrm{H}\right.$ ) reached the highest values at $18^{\circ} \mathrm{C}$ (Table 1 ), whereas the ratio between elemental composition and dry weight showed more constancy during the development at all three temperatures tested.

The elemental composition of the exuviae is given in Table 3. The exuviae were collected from mass cultures at $12^{\circ} \mathrm{C}$. Table 4 shows the loss in percent of $\mathrm{W}, \mathrm{C}, \mathrm{N}, \mathrm{H}$ and energy $\left(\mathrm{mJ} \times \mathrm{Ind}^{-1}\right)$ by the exuviae at the end of each nauplius stage, as well as the percentage loss on the growth rate during each single nauplius stage (data in brackets). The loss of dry weight and carbon at the end of the development of the naupliar stages decreased during development, whereas the nitrogen loss was highest in nauplius $V$. The energy content of the exuviae was 0.3 to $3.2 \mathrm{~mJ}$ (Table 3), which is $2-5 \%$ of the larval energy content (Table 4). The exuviae of the cypris larvae have a higher energy content $(6.9 \mathrm{~mJ})$, which is still a loss of only $4-7 \%$ of the total larval energy content. The energy loss by the exuviae on the growth rate of the single naupliar stages reached highest values in nauplius III (11-12\%) and IV (8-11\%), whereas the nauplius stage VI loses only $2-4 \%$ of its growth rate with the exuviae (Table 4 ). The lowest biomass loss by 
Table 1. Mean values of the biomass for the larval stages of Elminius modestus at 12,18 and $24{ }^{\circ} \mathrm{C}$. $\mathrm{W}$ : individual dry weight, $\mathrm{C}$ : carbon, $\mathrm{N}$ : nitrogen, $\mathrm{H}$ : hydrogen; $\overline{\mathrm{x}}$ : mean values, $\pm: 95 \%$ confidence interval; individual and weight specific energy content in Joule; $n$ : numbers of experiments, No.: numbers of individuals per experiment

\begin{tabular}{|c|c|c|c|c|c|c|c|}
\hline $\begin{array}{c}12^{\circ} \mathrm{C} \\
\text { Larval stage }\end{array}$ & & II & III & IV & $\mathrm{V}$ & VI & Cypris \\
\hline $\mathrm{W}(\mu \mathrm{g})$ & $\begin{array}{l}\bar{x} \\
\pm\end{array}$ & $\begin{array}{l}0.39 \\
0.03\end{array}$ & $\begin{array}{l}0.71 \\
0.04\end{array}$ & $\begin{array}{l}1.20 \\
0.08\end{array}$ & $\begin{array}{l}2.33 \\
0.32\end{array}$ & $\begin{array}{l}4.27 \\
0.17\end{array}$ & $\begin{array}{l}4.56 \\
0.48\end{array}$ \\
\hline $\mathrm{C}(\%)$ & $\begin{array}{l}\bar{x} \\
\pm\end{array}$ & $\begin{array}{c}40.77 \\
2.6\end{array}$ & $\begin{array}{r}41.33 \\
1.28\end{array}$ & $\begin{array}{r}43.92 \\
2.68\end{array}$ & $\begin{array}{r}37.79 \\
1.37\end{array}$ & $\begin{array}{r}39.57 \\
1.17\end{array}$ & $\begin{array}{r}51.78 \\
3.52\end{array}$ \\
\hline$C(\mu g)$ & $\begin{array}{l}\bar{x} \\
\pm\end{array}$ & $\begin{array}{l}0.15 \\
0.01\end{array}$ & $\begin{array}{l}0.29 \\
0.01\end{array}$ & $\begin{array}{l}0.51 \\
0.02\end{array}$ & $\begin{array}{l}0.87 \\
0.11\end{array}$ & $\begin{array}{l}1.76 \\
0.07\end{array}$ & $\begin{array}{l}2.30 \\
0.12\end{array}$ \\
\hline N (\%) & $\begin{array}{l}\overrightarrow{\mathbf{x}} \\
\pm\end{array}$ & $\begin{array}{r}10.68 \\
0.83\end{array}$ & $\begin{array}{r}10.14 \\
0.23\end{array}$ & $\begin{array}{r}11.29 \\
0.75\end{array}$ & $\begin{array}{l}9.51 \\
0.40\end{array}$ & $\begin{array}{l}9.05 \\
0.29\end{array}$ & $\begin{array}{r}10.45 \\
0.82\end{array}$ \\
\hline$N(\mu g)$ & $\begin{array}{l}\bar{x} \\
\pm\end{array}$ & $\begin{array}{l}0.04 \\
0.00\end{array}$ & $\begin{array}{l}0.07 \\
0.00\end{array}$ & $\begin{array}{l}0.13 \\
0.01\end{array}$ & $\begin{array}{l}0.22 \\
0.03\end{array}$ & $\begin{array}{l}0.39 \\
0.03\end{array}$ & $\begin{array}{l}0.45 \\
0.02\end{array}$ \\
\hline $\mathrm{H}(\%)$ & $\begin{array}{l}\bar{x} \\
\pm\end{array}$ & $\begin{array}{l}5.68 \\
0.37\end{array}$ & $\begin{array}{l}5.81 \\
0.23\end{array}$ & $\begin{array}{l}6.30 \\
0.30\end{array}$ & $\begin{array}{l}5.53 \\
0.19\end{array}$ & $\begin{array}{l}6.03 \\
0.18\end{array}$ & $\begin{array}{l}7.64 \\
0.36\end{array}$ \\
\hline $\mathrm{H}(\mu \mathrm{g})$ & $\begin{array}{l}\bar{x} \\
\pm\end{array}$ & $\begin{array}{l}0.02 \\
0.00\end{array}$ & $\begin{array}{l}0.04 \\
0.00\end{array}$ & $\begin{array}{l}0.07 \\
0.00\end{array}$ & $\begin{array}{l}0.13 \\
0.02\end{array}$ & $\begin{array}{l}0.27 \\
0.01\end{array}$ & $\begin{array}{l}0.35 \\
0.03\end{array}$ \\
\hline$C: N$ & $\begin{array}{l}\bar{x} \\
\pm\end{array}$ & $\begin{array}{l}3.84 \\
0.07\end{array}$ & $\begin{array}{l}4.08 \\
0.06\end{array}$ & $\begin{array}{l}3.89 \\
0.03\end{array}$ & $\begin{array}{l}3.97 \\
0.05\end{array}$ & $\begin{array}{l}4.36 \\
0.05\end{array}$ & $\begin{array}{l}4.95 \\
0.09\end{array}$ \\
\hline $\mathrm{C}: \mathrm{H}$ & $\begin{array}{l}\overline{\mathbf{x}} \\
\pm\end{array}$ & $\begin{array}{l}7.28 \\
0.35\end{array}$ & $\begin{array}{l}7.08 \\
0.11\end{array}$ & $\begin{array}{l}6.95 \\
0.15\end{array}$ & $\begin{array}{l}6.83 \\
0.15\end{array}$ & $\begin{array}{l}6.78 \\
0.14\end{array}$ & $\begin{array}{l}6.73 \\
0.17\end{array}$ \\
\hline $\mathrm{mJ} \times \mathrm{ind}^{-1}$ & $\begin{array}{l}\overline{\mathbf{x}} \\
\pm\end{array}$ & $\begin{array}{l}5.72 \\
0.43\end{array}$ & $\begin{array}{r}10.99 \\
3.10\end{array}$ & $\begin{array}{r}19.61 \\
0.93\end{array}$ & $\begin{array}{r}31.03 \\
4.07\end{array}$ & $\begin{array}{r}64.88 \\
2.70\end{array}$ & $\begin{array}{r}97.95 \\
4.91\end{array}$ \\
\hline $\mathrm{J} \times \mathrm{mg} \mathrm{W}^{-1}$ & $\begin{array}{l}\bar{x} \\
\pm\end{array}$ & $\begin{array}{r}15.30 \\
1.47\end{array}$ & $\begin{array}{r}15.36 \\
0.69\end{array}$ & $\begin{array}{r}17.01 \\
1.58\end{array}$ & $\begin{array}{r}13.50 \\
0.73\end{array}$ & $\begin{array}{r}15.03 \\
0.78\end{array}$ & $\begin{array}{r}21.48 \\
1.70\end{array}$ \\
\hline $\mathrm{n}$ & & 27 & 19 & 20 & 19 & 60 & 20 \\
\hline No. & & 1000 & 600 & 350 & 250 & 150 & 100 \\
\hline $\begin{array}{c}18^{\circ} \mathrm{C} \\
\text { Larval stage }\end{array}$ & & II & III & IV & v & $\mathrm{VI}$ & Cypris \\
\hline $\mathrm{W}(\mu \mathrm{g})$ & $\begin{array}{l}\bar{x} \\
\pm\end{array}$ & $\begin{array}{l}0.41 \\
0.03\end{array}$ & $\begin{array}{l}0.75 \\
0.07\end{array}$ & $\begin{array}{l}1.47 \\
0.15\end{array}$ & $\begin{array}{l}2.62 \\
0.18\end{array}$ & $\begin{array}{l}5.19 \\
0.18\end{array}$ & $\begin{array}{l}5.81 \\
0.27\end{array}$ \\
\hline $\mathrm{C}(\%)$ & $\begin{array}{l}\bar{x} \\
\pm\end{array}$ & $\begin{array}{r}42.10 \\
2.41\end{array}$ & $\begin{array}{r}40.97 \\
3.20\end{array}$ & $\begin{array}{r}37.09 \\
5.17\end{array}$ & $\begin{array}{r}40.23 \\
2.46\end{array}$ & $\begin{array}{r}43.95 \\
1.45\end{array}$ & $\begin{array}{r}56.56 \\
1.49\end{array}$ \\
\hline$C(\mu \mathrm{g})$ & $\begin{array}{l}\bar{x} \\
\pm\end{array}$ & $\begin{array}{l}0.17 \\
0.01\end{array}$ & $\begin{array}{l}0.31 \\
0.01\end{array}$ & $\begin{array}{l}0.54 \\
0.06\end{array}$ & $\begin{array}{l}1.05 \\
0.05\end{array}$ & $\begin{array}{l}2.17 \\
0.08\end{array}$ & $\begin{array}{l}3.30 \\
0.10\end{array}$ \\
\hline$N(\%)$ & $\begin{array}{l}\overline{\mathbf{x}} \\
\pm\end{array}$ & $\begin{array}{r}10.88 \\
0.61\end{array}$ & $\begin{array}{r}10.89 \\
0.94\end{array}$ & $\begin{array}{l}9.55 \\
0.59\end{array}$ & $\begin{array}{l}9.22 \\
0.55\end{array}$ & $\begin{array}{l}8.72 \\
0.28\end{array}$ & $\begin{array}{r}10.16 \\
0.26\end{array}$ \\
\hline$N(\mu \mathrm{g})$ & $\begin{array}{l}\overline{\mathbf{x}} \\
\pm\end{array}$ & $\begin{array}{l}0.04 \\
0.00\end{array}$ & $\begin{array}{l}0.08 \\
0.00\end{array}$ & $\begin{array}{l}0.14 \\
0.01\end{array}$ & $\begin{array}{l}0.23 \\
0.00\end{array}$ & $\begin{array}{l}0.43 \\
0.02\end{array}$ & $\begin{array}{l}0.59 \\
0.02\end{array}$ \\
\hline
\end{tabular}


Table 1 (continued)

\begin{tabular}{|c|c|c|c|c|c|c|c|}
\hline $\begin{array}{c}18^{\circ} \mathrm{C} \\
\text { Larval stage }\end{array}$ & & II & III & IV & v & VI & Cypris \\
\hline $\mathrm{H}(\%)$ & $\begin{array}{l}\overline{\mathbf{x}} \\
\pm\end{array}$ & $\begin{array}{l}6.14 \\
0.38\end{array}$ & $\begin{array}{l}5.85 \\
0.36\end{array}$ & $\begin{array}{l}5.83 \\
0.38\end{array}$ & $\begin{array}{l}6.14 \\
0.31\end{array}$ & $\begin{array}{l}6.60 \\
0.19\end{array}$ & $\begin{array}{l}8.31 \\
0.20\end{array}$ \\
\hline$H(\mu g)$ & $\begin{array}{l}\overline{\mathbf{x}} \\
\pm\end{array}$ & $\begin{array}{l}0.02 \\
0.00\end{array}$ & $\begin{array}{l}0.04 \\
0.00\end{array}$ & $\begin{array}{l}0.09 \\
0.01\end{array}$ & $\begin{array}{l}0.16 \\
0.01\end{array}$ & $\begin{array}{l}0.32 \\
0.01\end{array}$ & $\begin{array}{l}0.49 \\
0.02\end{array}$ \\
\hline$C: N$ & $\begin{array}{l}\bar{x} \\
\pm\end{array}$ & $\begin{array}{l}3.87 \\
0.06\end{array}$ & $\begin{array}{l}3.78 \\
0.04\end{array}$ & $\begin{array}{l}3.88 \\
0.09\end{array}$ & $\begin{array}{l}4.35 \\
0.03\end{array}$ & $\begin{array}{l}5.01 \\
0.04\end{array}$ & $\begin{array}{l}5.56 \\
0.03\end{array}$ \\
\hline $\mathrm{C}: \mathrm{H}$ & $\begin{array}{l}\bar{x} \\
\pm\end{array}$ & $\begin{array}{l}6.93 \\
0.39\end{array}$ & $\begin{array}{l}7.03 \\
0.49\end{array}$ & $\begin{array}{l}6.38 \\
0.19\end{array}$ & $\begin{array}{l}6.53 \\
0.21\end{array}$ & $\begin{array}{l}6.62 \\
0.08\end{array}$ & $\begin{array}{l}6.81 \\
0.07\end{array}$ \\
\hline $\mathrm{mJ} \times$ ind $^{-1}$ & $\begin{array}{l}\overline{\mathbf{x}} \\
\pm\end{array}$ & $\begin{array}{l}6.30 \\
0.28\end{array}$ & $\begin{array}{r}11.28 \\
0.81\end{array}$ & $\begin{array}{r}19.34 \\
2.50\end{array}$ & $\begin{array}{r}38.62 \\
2.31\end{array}$ & $\begin{array}{r}89.10 \\
2.90\end{array}$ & $\begin{array}{r}142.50 \\
4.43\end{array}$ \\
\hline $\mathrm{J} \times \mathrm{mg} \mathrm{W}^{-1}$ & $\begin{array}{l}\overline{\mathbf{x}} \\
\pm\end{array}$ & $\begin{array}{r}15.90 \\
1.32\end{array}$ & $\begin{array}{r}15.23 \\
1.73\end{array}$ & $\begin{array}{r}13.18 \\
1.33\end{array}$ & $\begin{array}{r}14.09 \\
1.27\end{array}$ & $\begin{array}{r}17.13 \\
0.85\end{array}$ & $\begin{array}{r}24.65 \\
0.99\end{array}$ \\
\hline $\mathbf{n}$ & & 27 & 13 & 13 & 20 & 39 & 22 \\
\hline No. & & 1000 & 600 & 350 & 250 & 150 & 100 \\
\hline $\begin{array}{c}24^{\circ} \mathrm{C} \\
\text { Larval stage } \\
\end{array}$ & & II & III & IV & v & $\mathrm{VI}$ & Cypris \\
\hline $\mathrm{W}(\mu \mathrm{g})$ & $\begin{array}{l}\overline{\mathbf{x}} \\
\pm\end{array}$ & $\begin{array}{l}0.39 \\
0.03\end{array}$ & $\begin{array}{l}0.70 \\
0.14\end{array}$ & $\begin{array}{l}1.06 \\
0.10\end{array}$ & $\begin{array}{l}2.45 \\
0.16\end{array}$ & $\begin{array}{l}4.39 \\
0.75\end{array}$ & $\begin{array}{l}4.38 \\
0.28\end{array}$ \\
\hline$C(\%)$ & $\begin{array}{l}\bar{x} \\
\pm\end{array}$ & $\begin{array}{r}47.08 \\
2.13\end{array}$ & $\begin{array}{r}50.22 \\
4.99\end{array}$ & $\begin{array}{r}40.19 \\
2.28\end{array}$ & $\begin{array}{r}40.09 \\
2.62\end{array}$ & $\begin{array}{r}50.56 \\
6.71\end{array}$ & $\begin{array}{r}47.49 \\
1.79\end{array}$ \\
\hline$C(\mu \mathrm{g})$ & $\begin{array}{l}\overline{\mathrm{x}} \\
\pm\end{array}$ & $\begin{array}{l}0.18 \\
0.01\end{array}$ & $\begin{array}{l}0.34 \\
0.04\end{array}$ & $\begin{array}{l}0.43 \\
0.02\end{array}$ & $\begin{array}{l}0.68 \\
0.06\end{array}$ & $\begin{array}{l}2.13 \\
1.34\end{array}$ & $\begin{array}{l}2.07 \\
0.33\end{array}$ \\
\hline $\mathrm{N}(\%)$ & $\begin{array}{l}\bar{x} \\
\pm\end{array}$ & $\begin{array}{r}11.96 \\
0.63\end{array}$ & $\begin{array}{r}12.76 \\
1.24\end{array}$ & $\begin{array}{r}10.25 \\
0.83\end{array}$ & $\begin{array}{l}9.25 \\
0.58\end{array}$ & $\begin{array}{r}10.62 \\
1.34\end{array}$ & $\begin{array}{l}8.81 \\
0.33\end{array}$ \\
\hline$N(\mu g)$ & $\begin{array}{l}\bar{x} \\
\pm\end{array}$ & $\begin{array}{l}0.05 \\
0.00\end{array}$ & $\begin{array}{l}0.09 \\
0.01\end{array}$ & $\begin{array}{l}0.11 \\
0.01\end{array}$ & $\begin{array}{l}0.16 \\
0.01\end{array}$ & $\begin{array}{l}0.45 \\
0.05\end{array}$ & $\begin{array}{l}0.39 \\
0.02\end{array}$ \\
\hline $\mathrm{H}(\%)$ & $\begin{array}{l}\overline{\mathrm{x}} \\
\pm\end{array}$ & $\begin{array}{l}6.59 \\
0.25\end{array}$ & $\begin{array}{l}6.77 \\
0.71\end{array}$ & $\begin{array}{l}5.83 \\
0.38\end{array}$ & $\begin{array}{l}5.72 \\
0.30\end{array}$ & $\begin{array}{l}6.98 \\
0.82\end{array}$ & $\begin{array}{l}6.69 \\
0.23\end{array}$ \\
\hline $\mathrm{H}(\mu \mathrm{g})$ & $\begin{array}{l}\overline{\mathbf{x}} \\
\pm\end{array}$ & $\begin{array}{l}0.03 \\
0.00\end{array}$ & $\begin{array}{l}0.05 \\
0.01\end{array}$ & $\begin{array}{l}0.06 \\
0.00\end{array}$ & $\begin{array}{l}0.10 \\
0.01\end{array}$ & $\begin{array}{l}0.29 \\
0.04\end{array}$ & $\begin{array}{l}0.29 \\
0.02\end{array}$ \\
\hline$C: N$ & $\begin{array}{l}\bar{x} \\
\pm\end{array}$ & $\begin{array}{l}3.94 \\
0.05\end{array}$ & $\begin{array}{l}3.94 \\
0.08\end{array}$ & $\begin{array}{l}3.74 \\
0.20\end{array}$ & $\begin{array}{l}4.32 \\
0.11\end{array}$ & $\begin{array}{l}4.67 \\
0.15\end{array}$ & $\begin{array}{l}5.34 \\
0.12\end{array}$ \\
\hline $\mathrm{C}: \mathrm{H}$ & $\begin{array}{l}\overline{\mathbf{x}} \\
\pm\end{array}$ & $\begin{array}{l}7.14 \\
0.25\end{array}$ & $\begin{array}{l}7.43 \\
0.22\end{array}$ & $\begin{array}{l}6.87 \\
0.23\end{array}$ & $\begin{array}{l}6.97 \\
0.15\end{array}$ & $\begin{array}{l}7.33 \\
0.23\end{array}$ & $\begin{array}{l}7.07 \\
0.07\end{array}$ \\
\hline $\mathrm{mJ} \times$ ind $^{-1}$ & $\begin{array}{l}\bar{x} \\
\pm\end{array}$ & $\begin{array}{l}7.28 \\
0.41\end{array}$ & $\begin{array}{r}14.13 \\
1.85\end{array}$ & $\begin{array}{r}15.82 \\
0.84\end{array}$ & $\begin{array}{r}35.69 \\
3.45\end{array}$ & $\begin{array}{l}90.20 \\
14.02\end{array}$ & $\begin{array}{r}83.13 \\
5.25\end{array}$ \\
\hline $\mathrm{J} \times \mathrm{mg} \mathrm{W}^{-1}$ & $\begin{array}{l}\overline{\mathbf{x}} \\
\pm\end{array}$ & $\begin{array}{r}18.78 \\
1.22\end{array}$ & $\begin{array}{r}20.63 \\
3.03\end{array}$ & $\begin{array}{r}15.01 \\
1.33\end{array}$ & $\begin{array}{r}14.83 \\
1.42\end{array}$ & $\begin{array}{r}21.92 \\
4.55\end{array}$ & $\begin{array}{r}19.09 \\
1.07\end{array}$ \\
\hline $\mathrm{n}$ & & 26 & 8 & 15 & 23 & 10 & 28 \\
\hline No. & & 1000 & 450 & 350 & 250 & 150 & 100 \\
\hline
\end{tabular}


Table 2. Constants for the exponential equation $(\ln y=b+m t)$ for the calculation of the individual dry weight (W), content of carbon $(\mathrm{C})$, nitrogen $(\mathrm{N})$ and hydrogen $(\mathrm{H})$ as well as for the individual energy content $\left(\mathrm{mJ} \times\right.$ ind $\left.^{-1}\right)$ at 12,18 and $24^{\circ} \mathrm{C}$. The correlation coefficient lies between $0.985-0.996$ which is a confidence interval of $p<0.01-0.001$

\begin{tabular}{|cccccccc|}
\hline Constants & $\mathrm{b}$ & $\mathrm{m}$ & $\mathrm{b}$ & $\mathrm{m}$ & $\mathrm{b}$ & $\mathrm{m}$ \\
\hline & \multicolumn{2}{c}{$12^{\circ} \mathrm{C}$} & \multicolumn{2}{c}{$18^{\circ} \mathrm{C}$} & \multicolumn{2}{c}{$24^{\circ} \mathrm{C}$} \\
$\mathrm{W}$ & 0.31 & 0.20 & 0.34 & 0.33 & 0.30 & 0.52 \\
$\mathrm{C}$ & 0.13 & 0.20 & 0.13 & 0.34 & 0.13 & 0.50 \\
$\mathrm{~N}$ & 0.03 & 0.19 & 0.04 & 0.30 & 0.04 & 0.50 \\
$\mathrm{H}$ & 0.02 & 0.21 & 0.02 & 0.34 & 0.02 & 0.50 \\
$\mathrm{~mJ}$ ind $^{-1}$ & 4.75 & 0.20 & 4.80 & 0.35 & 5.19 & 0.52 \\
\hline
\end{tabular}

Table 3. Biomass values for the exuviae of the larval stages of Elminius modestus at $12^{\circ} \mathrm{C}$ (for further information see Table 1)

\begin{tabular}{|c|c|c|c|c|c|c|c|}
\hline Larval stage & & II & III & IV & V & VI & Cypris \\
\hline $\mathrm{W}(\mu \mathrm{g})$ & $\begin{array}{l}\bar{x} \\
\pm\end{array}$ & $\begin{array}{l}0.034 \\
0.007\end{array}$ & $\begin{array}{l}0.059 \\
0.017\end{array}$ & $\begin{array}{l}0.067 \\
0.020\end{array}$ & $\begin{array}{l}0.145 \\
0.026\end{array}$ & $\begin{array}{l}0.236 \\
0.066\end{array}$ & $\begin{array}{l}0.311 \\
0.012\end{array}$ \\
\hline$C(\%)$ & $\begin{array}{l}\overline{\mathbf{x}} \\
\pm\end{array}$ & $\begin{array}{r}31.33 \\
5.38\end{array}$ & $\begin{array}{r}28.08 \\
4.96\end{array}$ & $\begin{array}{r}38.29 \\
9.68\end{array}$ & $\begin{array}{r}37.76 \\
4.52\end{array}$ & $\begin{array}{l}41.77 \\
10.63\end{array}$ & $\begin{array}{r}53.18 \\
5.74\end{array}$ \\
\hline$C(\mu \mathrm{g})$ & $\begin{array}{l}\overline{\mathbf{x}} \\
\pm\end{array}$ & $\begin{array}{l}0.010 \\
0.001\end{array}$ & $\begin{array}{l}0.017 \\
0.001\end{array}$ & $\begin{array}{l}0.023 \\
0.002\end{array}$ & $\begin{array}{l}0.053 \\
0.004\end{array}$ & $\begin{array}{l}0.091 \\
0.005\end{array}$ & $\begin{array}{l}0.160 \\
0.011\end{array}$ \\
\hline N (\%) & $\begin{array}{l}\overline{\mathbf{x}} \\
\pm\end{array}$ & $\begin{array}{l}2.74 \\
1.45\end{array}$ & $\begin{array}{l}2.12 \\
0.37\end{array}$ & $\begin{array}{l}4.22 \\
0.88\end{array}$ & $\begin{array}{l}9.06 \\
1.00\end{array}$ & $\begin{array}{l}7.16 \\
1.86\end{array}$ & $\begin{array}{r}11.71 \\
1.17\end{array}$ \\
\hline$N(\mu g)$ & $\begin{array}{l}\overline{\mathbf{x}} \\
\pm\end{array}$ & $\begin{array}{l}0.001 \\
0.000\end{array}$ & $\begin{array}{l}0.002 \\
0.000\end{array}$ & $\begin{array}{l}0.003 \\
0.000\end{array}$ & $\begin{array}{l}0.012 \\
0.000\end{array}$ & $\begin{array}{l}0.016 \\
0.001\end{array}$ & $\begin{array}{l}0.036 \\
0.002\end{array}$ \\
\hline$H(\%)$ & $\begin{array}{l}\overline{\mathbf{x}} \\
\pm\end{array}$ & $\begin{array}{l}3.74 \\
0.99\end{array}$ & $\begin{array}{l}3.23 \\
0.36\end{array}$ & $\begin{array}{l}4.47 \\
0.74\end{array}$ & $\begin{array}{l}5.05 \\
0.59\end{array}$ & $\begin{array}{l}6.05 \\
1.41\end{array}$ & $\begin{array}{l}6.15 \\
0.57\end{array}$ \\
\hline $\mathrm{H}(\mu \mathrm{g})$ & $\begin{array}{l}\overrightarrow{\mathbf{x}} \\
\pm\end{array}$ & $\begin{array}{l}0.001 \\
0.000\end{array}$ & $\begin{array}{l}0.002 \\
0.001\end{array}$ & $\begin{array}{l}0.003 \\
0.001\end{array}$ & $\begin{array}{l}0.007 \\
0.001\end{array}$ & $\begin{array}{l}0.013 \\
0.001\end{array}$ & $\begin{array}{l}0.019 \\
0.001\end{array}$ \\
\hline$C: N$ & $\begin{array}{l}\overline{\mathbf{x}} \\
\pm\end{array}$ & $\begin{array}{r}14.44 \\
5.93\end{array}$ & $\begin{array}{r}13.34 \\
3.86\end{array}$ & $\begin{array}{l}9.06 \\
1.08\end{array}$ & $\begin{array}{l}4.17 \\
0.16\end{array}$ & $\begin{array}{l}5.84 \\
0.16\end{array}$ & $\begin{array}{l}4.54 \\
0.03\end{array}$ \\
\hline $\mathrm{C}: \mathrm{H}$ & $\begin{array}{l}\overrightarrow{\mathbf{x}} \\
\pm\end{array}$ & $\begin{array}{l}8.82 \\
1.91\end{array}$ & $\begin{array}{l}8.81 \\
1.79\end{array}$ & $\begin{array}{l}8.45 \\
0.98\end{array}$ & $\begin{array}{l}7.49 \\
0.55\end{array}$ & $\begin{array}{l}6.88 \\
0.38\end{array}$ & $\begin{array}{l}8.63 \\
0.13\end{array}$ \\
\hline $\mathrm{mJ} \times$ exuviae $^{-1}$ & $\begin{array}{l}\bar{x} \\
\pm\end{array}$ & $\begin{array}{l}0.34 \\
0.04\end{array}$ & $\begin{array}{l}0.54 \\
0.06\end{array}$ & $\begin{array}{l}0.84 \\
0.07\end{array}$ & $\begin{array}{l}1.88 \\
0.11\end{array}$ & $\begin{array}{l}3.21 \\
0.33\end{array}$ & $\begin{array}{l}6.95 \\
0.88\end{array}$ \\
\hline $\mathrm{J} \times \mathrm{mg} \mathrm{W}^{-1}$ & $\begin{array}{l}\bar{x} \\
\pm\end{array}$ & $\begin{array}{r}10.42 \\
2.47\end{array}$ & $\begin{array}{l}8.94 \\
2.12\end{array}$ & $\begin{array}{r}14.23 \\
5.33\end{array}$ & $\begin{array}{r}13.56 \\
2.27\end{array}$ & $\begin{array}{r}16.16 \\
6.25\end{array}$ & $\begin{array}{r}22.47 \\
3.69\end{array}$ \\
\hline $\mathrm{n}$ & & 8 & 8 & 8 & 9 & 8 & 8 \\
\hline No. & & 1000 & 1000 & 1000 & 800 & 500 & 300 \\
\hline
\end{tabular}


Table 4. Percentage of biomass loss by the exuviae at the time of moulting and of the growth rate during each nauplius stage (data in brackets) $\left[\mathrm{W}=\mathrm{dry}_{\text {weight; }} \mathrm{C}, \mathrm{N}, \mathrm{H}=\right.$ content of carbon, nitrogen and hydrogen; $\mathrm{mJ} \times$ ind $^{-1}$. =energy content per individual]

\begin{tabular}{|c|c|c|c|c|c|c|c|}
\hline${ }^{\circ} \mathrm{C}$ & Larval stage & II & III & IV & v & $\mathrm{VI}$ & Cypris \\
\hline \multirow[t]{5}{*}{12} & w & $\begin{array}{c}10.3 \\
(13.1)\end{array}$ & $\begin{array}{c}6.6 \\
(18.4)\end{array}$ & $\begin{array}{r}4.7 \\
(12.6)\end{array}$ & $\begin{array}{r}5.2 \\
(10.7)\end{array}$ & $\begin{array}{c}2.8 \\
(4.3)\end{array}$ & 6.8 \\
\hline & C & $\begin{array}{c}4.4 \\
(9.8)\end{array}$ & $\begin{array}{r}4.7 \\
(13.1)\end{array}$ & $\begin{array}{c}4.1 \\
(10.9)\end{array}$ & $\begin{array}{c}4.7 \\
(9.7)\end{array}$ & $\begin{array}{c}2.7 \\
(4.1)\end{array}$ & 7.2 \\
\hline & $\mathrm{N}$ & $\begin{array}{c}1.5 \\
(3.5)\end{array}$ & $\begin{array}{c}1.6 \\
(4.8)\end{array}$ & $\begin{array}{c}1.8 \\
(5.1)\end{array}$ & $\begin{array}{c}4.5 \\
(9.7)\end{array}$ & $\begin{array}{c}2.1 \\
(3.2)\end{array}$ & 7.9 \\
\hline & $\mathrm{H}$ & $\begin{array}{c}3.3 \\
(6.8)\end{array}$ & $\begin{array}{c}3.8 \\
(10.5)\end{array}$ & $\begin{array}{c}3.3 \\
(8.7)\end{array}$ & $\begin{array}{c}4.3 \\
(8.7)\end{array}$ & $\begin{array}{c}2.6 \\
(3.8)\end{array}$ & 5.5 \\
\hline & $\mathrm{mJ} \times$ ind $^{-1}$ & $\begin{array}{c}3.9 \\
(8.7)\end{array}$ & $\begin{array}{c}4.0 \\
(11.4)\end{array}$ & $\begin{array}{c}3.9 \\
(10.7)\end{array}$ & $\begin{array}{c}4.6 \\
(9.4)\end{array}$ & $\begin{array}{c}2.6 \\
(4.0)\end{array}$ & 7.1 \\
\hline \multirow[t]{5}{*}{18} & $w$ & $\begin{array}{c}5.1 \\
(10.6)\end{array}$ & $\begin{array}{r}6.2 \\
(20.3)\end{array}$ & $\begin{array}{c}4.7 \\
(14.2)\end{array}$ & $\begin{array}{c}4.2 \\
(7.1)\end{array}$ & $\begin{array}{c}2.0 \\
(2.9)\end{array}$ & 5.3 \\
\hline & $\mathrm{C}$ & $\begin{array}{c}3.9 \\
(7.7)\end{array}$ & $\begin{array}{r}4.5 \\
(14.5)\end{array}$ & $\begin{array}{c}4.1 \\
(12.4)\end{array}$ & $\begin{array}{c}3.8 \\
(6.4)\end{array}$ & $\begin{array}{c}1.9 \\
(2.6)\end{array}$ & 5.0 \\
\hline & $\mathrm{N}$ & $\begin{array}{c}1.2 \\
(2.5)\end{array}$ & $\begin{array}{c}1.4 \\
(4.5)\end{array}$ & $\begin{array}{c}1.6 \\
(4.9)\end{array}$ & $\begin{array}{c}3.1 \\
(5.1)\end{array}$ & $\begin{array}{c}1.1 \\
(1.6)\end{array}$ & 6.1 \\
\hline & $\mathrm{H}$ & $\begin{array}{c}2.9 \\
(5.8)\end{array}$ & $\begin{array}{c}3.6 \\
(11.7)\end{array}$ & $\begin{array}{c}3.4 \\
(10.1)\end{array}$ & $\begin{array}{c}3.5 \\
(5.9)\end{array}$ & $\begin{array}{c}1.9 \\
(2.6)\end{array}$ & 3.9 \\
\hline & $\mathrm{mJ} \times$ ind $^{-1}$ & $\begin{array}{c}3.5 \\
(7.1)\end{array}$ & $\begin{array}{c}3.8 \\
(12.1)\end{array}$ & $\begin{array}{c}3.9 \\
(11.5)\end{array}$ & $\begin{array}{c}3.4 \\
(5.7)\end{array}$ & $\begin{array}{c}1.8 \\
(2.6)\end{array}$ & 4.9 \\
\hline \multirow[t]{5}{*}{24} & w & $\begin{array}{c}5.8 \\
(11.8)\end{array}$ & $\begin{array}{c}7.0 \\
(23.8)\end{array}$ & $\begin{array}{c}4.7 \\
(11.7)\end{array}$ & $\begin{array}{r}5.2 \\
(10.6)\end{array}$ & $\begin{array}{c}2.7 \\
(3.9)\end{array}$ & 7.1 \\
\hline & $\mathrm{C}$ & $\begin{array}{c}4.1 \\
(8.6)\end{array}$ & $\begin{array}{c}4.8 \\
(16.3)\end{array}$ & $\begin{array}{c}4.0 \\
(10.3)\end{array}$ & $\begin{array}{c}4.8 \\
(10.0)\end{array}$ & $\begin{array}{c}2.7 \\
(4.1)\end{array}$ & 7.9 \\
\hline & $\mathbf{N}$ & $\begin{array}{c}1.4 \\
(3.1)\end{array}$ & $\begin{array}{c}1.7 \\
(6.2)\end{array}$ & $\begin{array}{c}1.9 \\
(5.2)\end{array}$ & $\begin{array}{c}4.8 \\
(10.7)\end{array}$ & $\begin{array}{c}2.3 \\
(3.6)\end{array}$ & 9.3 \\
\hline & $\mathrm{H}$ & $\begin{array}{c}3.1 \\
(6.6)\end{array}$ & $\begin{array}{c}4.1 \\
(14.3)\end{array}$ & $\begin{array}{c}3.4 \\
(8.6)\end{array}$ & $\begin{array}{c}4.6 \\
(9.8)\end{array}$ & $\begin{array}{c}2.8 \\
(4.2)\end{array}$ & 6.5 \\
\hline & $\mathrm{mJ} \times \mathrm{ind}^{-1}$ & $\begin{array}{c}3.3 \\
(6.7)\end{array}$ & $\begin{array}{c}3.7 \\
(11.9)\end{array}$ & $\begin{array}{c}3.4 \\
(8.3)\end{array}$ & $\begin{array}{c}3.8 \\
(7.7)\end{array}$ & $\begin{array}{c}2.1 \\
(3.0)\end{array}$ & 6.6 \\
\hline
\end{tabular}

the exuviae was found at $18^{\circ} \mathrm{C}$. This indicates that at this temperature a higher percentage of the accumulated energy is converted into growth of the larvae than at $12^{\circ} \mathrm{C}$ and $24^{\circ} \mathrm{C}$.

\section{Respiration}

The individual respiration rate (ng $\mathrm{O}_{2} \times \operatorname{Ind}^{-1} \times \mathrm{h}^{-1}$ ) increased with the larval development up to the VIth nauplius stage, whereas the cypris showed a reduced respiration rate (Table 5). Table 5 shows further the energy equivalents and the weight specific respiration rate (mean dry weights of the single nauplius stages are calculated 
Table 5. Individual (ng $\mathrm{O}_{2} \times \mathrm{h}^{-1}$ ind $\mathrm{d}^{-1}$ ) and weight-specific respiration rate ( $\mathrm{ng} \mathrm{O}_{2} \times \mu \mathrm{g} \mathrm{W}^{-1} \mathrm{~h}^{-1}$ ) as well as the energy loss by metabolism $\left(\mathrm{M}=\mathrm{mJ}^{\circ} \mathrm{h}^{-1}\right)$ for the larval stages of Elminius modestus at 12,18 and $24^{\circ} \mathrm{C}(\overline{\mathrm{x}}$ mean value $\pm 95 \%$ confidence interval)

\begin{tabular}{|c|c|c|c|c|c|c|c|c|c|c|}
\hline \multirow{2}{*}{\multicolumn{2}{|c|}{ Larval stage }} & \multicolumn{3}{|c|}{ ng $\mathrm{O}_{2} \times \mathrm{h}^{-1}$ ind $^{-1}$} & \multicolumn{3}{|c|}{$\mathrm{ng} \mathrm{O}_{2} \times \mu \mathrm{g} \mathrm{W}^{-1} \mathrm{~h}^{-1}$} & \multicolumn{3}{|c|}{$M$} \\
\hline & & 12 & 18 & $24^{\circ} \mathrm{C}$ & 12 & 18 & $24^{\circ} \mathrm{C}$ & 12 & 18 & $24^{\circ} \mathrm{C}$ \\
\hline $\begin{array}{l}\text { II (newly } \\
\text { hatched) }\end{array}$ & $\begin{array}{l}\overline{\mathbf{x}} \\
\pm\end{array}$ & $\begin{array}{l}1.04 \\
0.14\end{array}$ & $\begin{array}{l}1.89 \\
0.14\end{array}$ & $\begin{array}{l}3.24 \\
0.15\end{array}$ & 3.21 & 5.82 & 9.98 & 0.015 & 0.027 & 0.046 \\
\hline II & $\begin{array}{l}\overline{\mathbf{x}} \\
\pm\end{array}$ & $\begin{array}{l}1.35 \\
0.15\end{array}$ & $\begin{array}{l}3.33 \\
0.29\end{array}$ & $\begin{array}{l}4.09 \\
0.62\end{array}$ & 3.45 & 8.12 & 10.47 & 0.019 & 0.047 & 0.057 \\
\hline III & $\begin{array}{l}\bar{x} \\
\pm\end{array}$ & $\begin{array}{l}2.39 \\
0.19\end{array}$ & $\begin{array}{l}5.17 \\
0.64\end{array}$ & $\begin{array}{l}7.70 \\
0.63\end{array}$ & 3.37 & 6.89 & 11.00 & 0.034 & 0.073 & 0.108 \\
\hline IV & $\begin{array}{l}\bar{x} \\
\pm\end{array}$ & $\begin{array}{l}4.20 \\
0.48\end{array}$ & $\begin{array}{r}10.98 \\
0.21\end{array}$ & $\begin{array}{r}15.13 \\
1.05\end{array}$ & 3.50 & 7.47 & 14.27 & 0.060 & 0.154 & 0.213 \\
\hline V & $\begin{array}{l}\bar{x} \\
\pm\end{array}$ & $\begin{array}{l}7.63 \\
0.48\end{array}$ & $\begin{array}{r}14.21 \\
1.79\end{array}$ & $\begin{array}{r}23.37 \\
2.06\end{array}$ & 3.27 & 5.42 & 9.53 & 0.107 & 0.200 & 0.328 \\
\hline VI & $\begin{array}{l}\bar{x} \\
\pm\end{array}$ & $\begin{array}{r}10.40 \\
1.34\end{array}$ & $\begin{array}{r}17.29 \\
0.52\end{array}$ & $\begin{array}{r}26.20 \\
2.24\end{array}$ & 2.44 & 3.33 & 5.97 & 0.146 & 0.243 & 0.368 \\
\hline Cypris & $\begin{array}{l}\bar{x} \\
\pm\end{array}$ & $\begin{array}{l}9.70 \\
0.87\end{array}$ & $\begin{array}{r}14.31 \\
1.44\end{array}$ & $\begin{array}{r}19.85 \\
1.43\end{array}$ & 2.12 & 2.46 & 2.82 & 0.136 & 0.201 & 0.279 \\
\hline
\end{tabular}

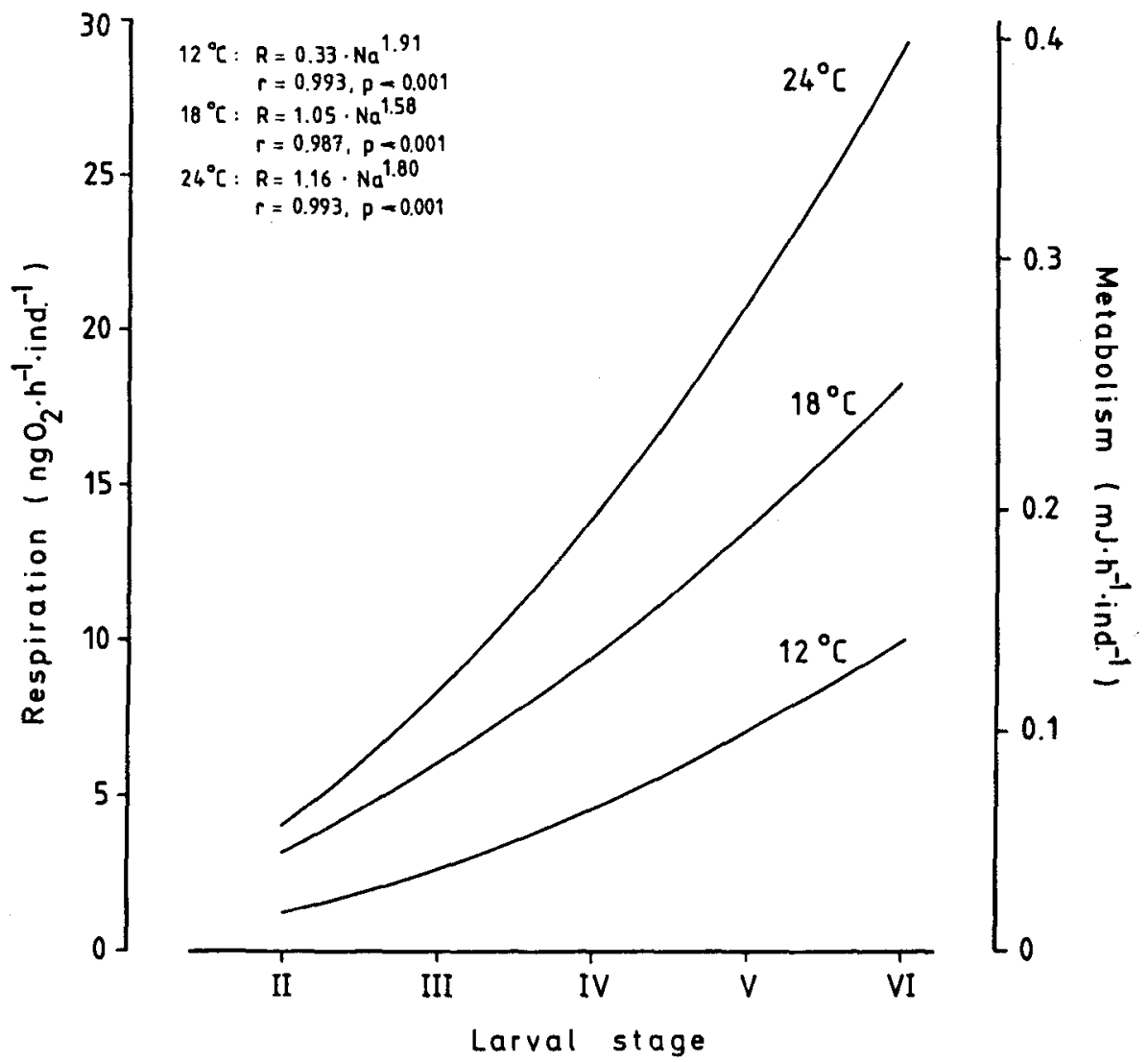

Fig. 1. Fittings for the increase of the respiration rate and metabolism during development from nauplius II to the VI. nauplius stage of Elminius modestus. $\mathrm{R}=$ respiration rate; $\mathrm{Na}=$ nauplius staren. $r=$ enrrelation conffirient: $n=$ ronfidence interval 
from Table 2). The increase of the respiration rate with the larval stages could be expressed by a power function (Fig. 1):

$$
\mathrm{R}=\mathrm{b} \cdot \mathrm{Na}^{\mathrm{m}},
$$

$\mathrm{R}=$ respiration rate, $\mathrm{Na}=$ nauplius stage, $\mathrm{b}$ and $\mathrm{m}=$ constants.

The increase of the respiration rate during the development time could also be expressed by a power function (Fig. 2), but increased logarithmically with the dry weight of the larvae (Fig. 3).

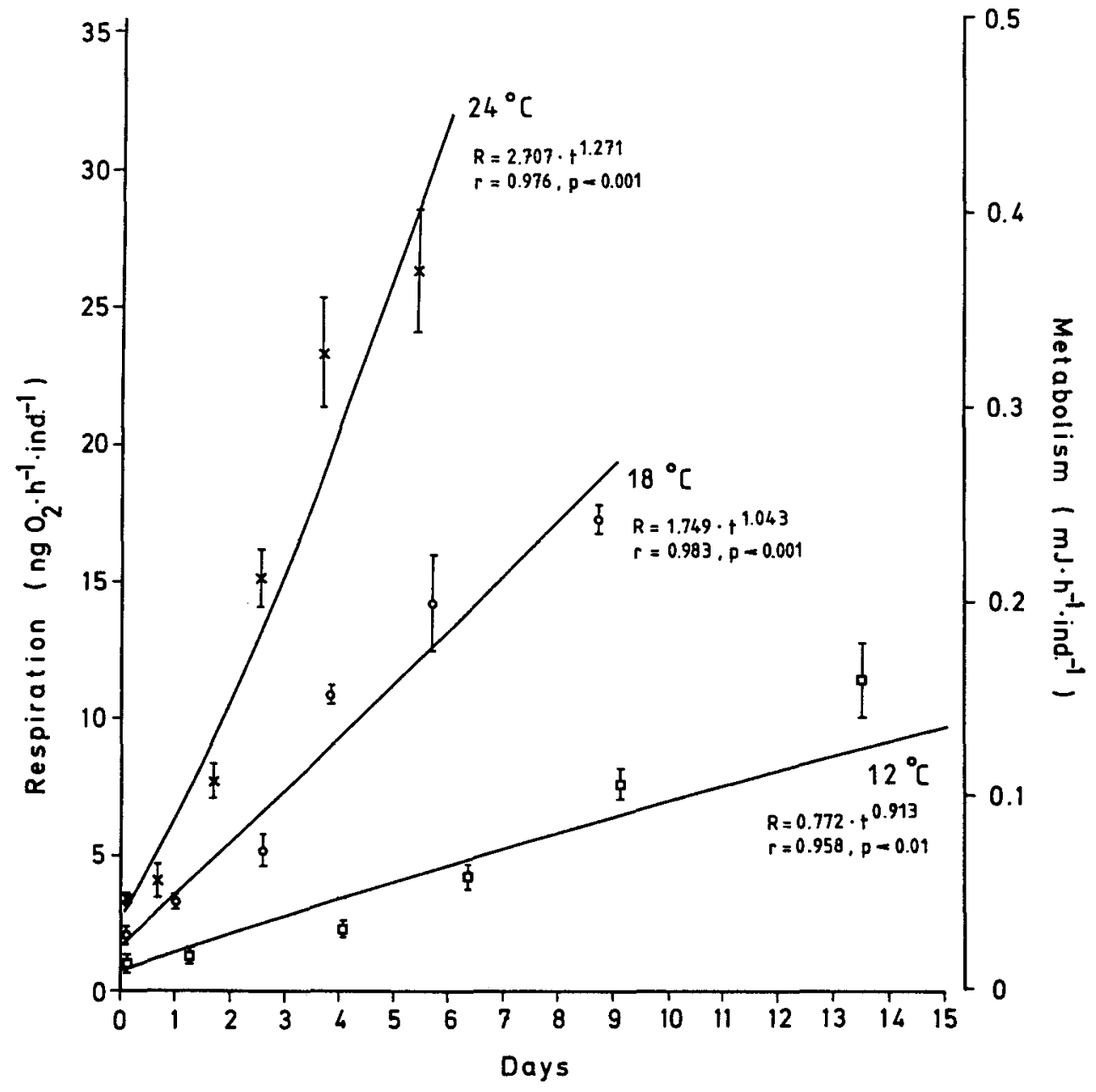

Fig. 2. Fittings for the increase of the respiration rate and metabolism during the duration of larval development of Elminius modestus. $\mathrm{R}=$ respiration rate; $\mathrm{t}=$ developmental duration; $\mathrm{r}=$ correlation coefficient $; p=$ confidence interval 


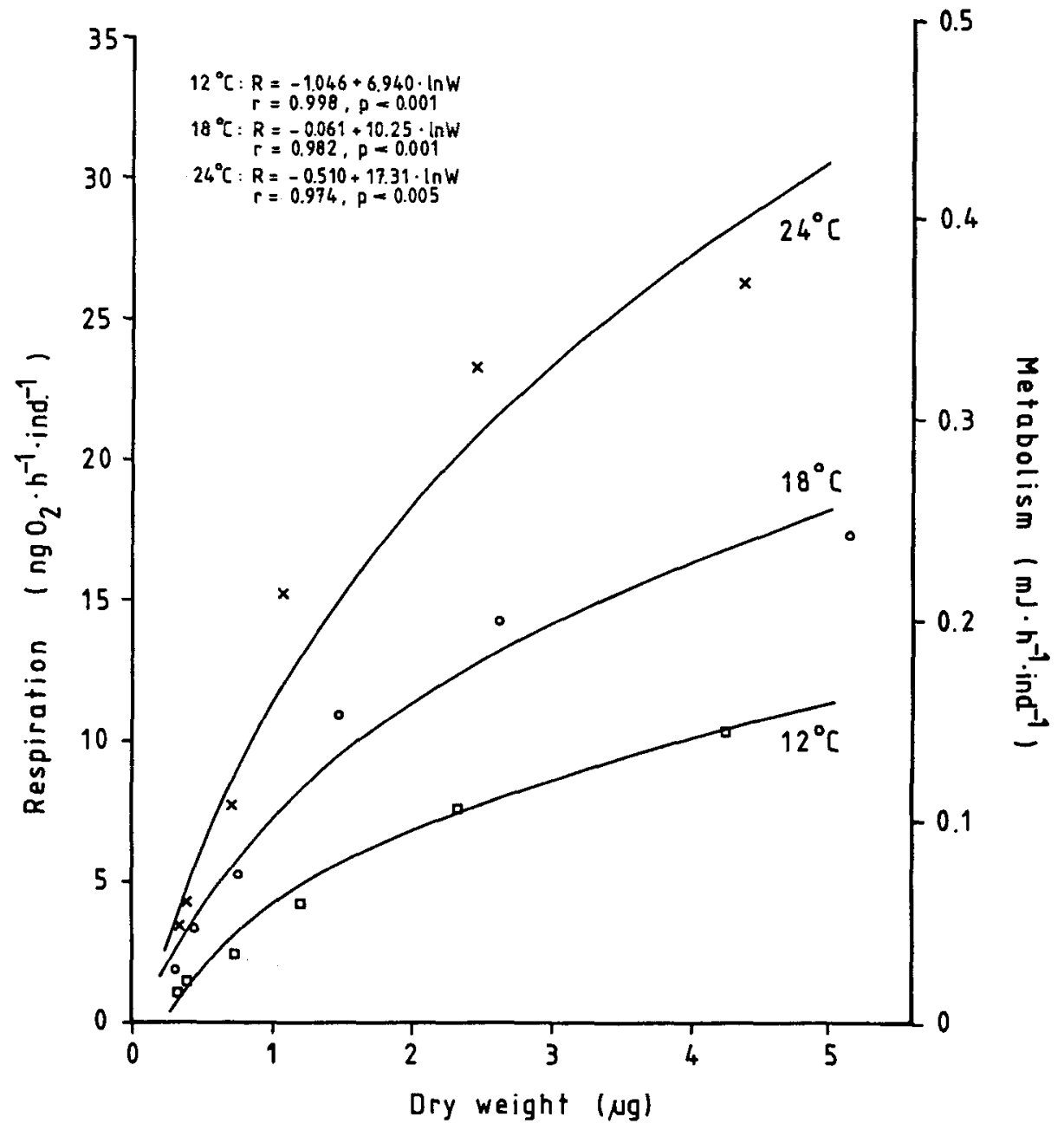

Fig. 3. Increase of respiration rate and metabolism with the dry weight of the larvae of Elminius modestus. $\mathrm{R}=$ respiration rate; $\mathrm{W}=$ dry weight; $\mathrm{r}=$ correlation coefficient; $\mathbf{p}=$ confidence interval

\section{Ingestion rate}

The number of cells of Skeletonema costatum ingested per day by the naupliar stages of Elminius modestus are given in Table 6 . The nauplius stage I and the cypris have not been considered, because they do not feed. The food uptake increased generally with temperature and developmental stage, except for stage VI $\left(12^{\circ} \mathrm{C}\right)$ and stage $\mathrm{V}\left(24^{\circ} \mathrm{C}\right)$. The increase of food uptake during development could be expressed by an exponential function at 12 and $18^{\circ} \mathrm{C}$ :

$$
\ln \mathrm{I}=\mathrm{b}+\mathrm{m} \mathrm{Na} \text {, }
$$


and logarithmically at $24^{\circ} \mathrm{C}$ (Fig. 4):

$$
\mathrm{I}=\operatorname{lnb}+\mathrm{m} \ln \mathrm{Na},
$$

$\mathrm{I}=$ ingestion rate, $\mathrm{Na}=$ nauplius stage, $\mathrm{b}$ and $\mathrm{m}=$ constants. The shift of the food uptake from an exponential to a logarithmical relationship indicates that the relative ingestion rate decreases at $24^{\circ} \mathrm{C}$. This might be a signal that $24^{\circ} \mathrm{C}$ is already a suboptimal temperature for the larval development of $E$. modestus.

The filtration rates increase with water temperature, but the values fluctuate during development and do not show a clear trend (Harms, 1985).

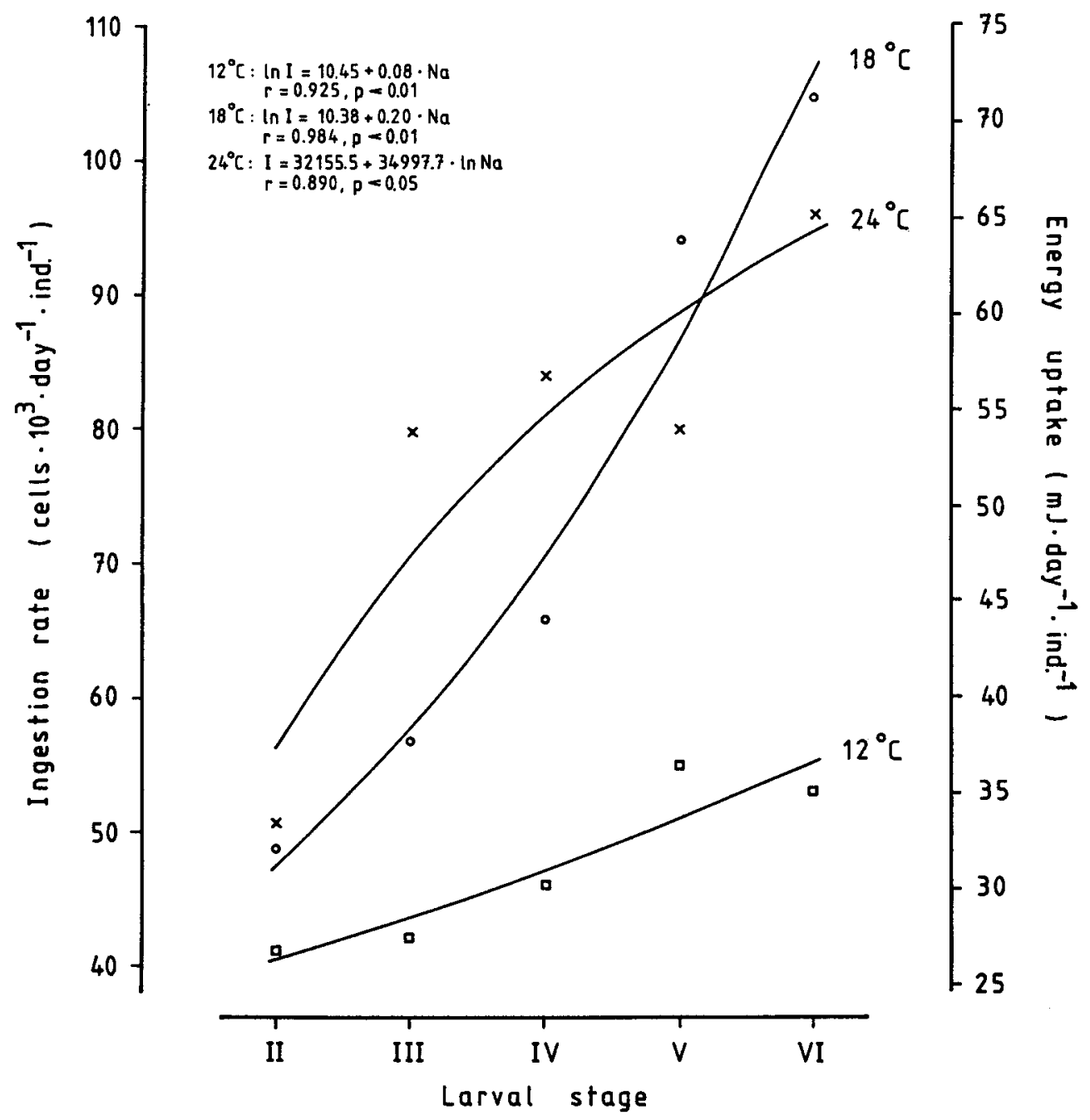

Fig. 4. Fitted ingestion rates for the nauplius stages of Elminius modestus. $\mathrm{I}=$ Ingestion rate $; \mathrm{Na}=$ nauplius stage $\mathrm{r}=$ correlation coefficient; $\mathrm{p}=$ confidence interval 
Table 6. Ingestion rate (cells/day) for the larval stages of Elminius modestus ( $\bar{x}$ mean value \pm standard deviation)

\begin{tabular}{|c|c|c|c|c|c|c|}
\hline \multicolumn{2}{|c|}{ Larval stage } & \multirow{2}{*}{$\begin{array}{c}\text { II } \\
41294 \\
14169\end{array}$} & \multirow{2}{*}{$\frac{\text { III }}{42049}$} & \multirow{2}{*}{$\begin{array}{r}\text { IV } \\
\begin{array}{r}46145 \\
2530\end{array}\end{array}$} & \multirow{2}{*}{$\begin{array}{r}\mathrm{V} \\
55326 \\
5837\end{array}$} & \multirow{2}{*}{$\begin{array}{c}\mathrm{VI} \\
\begin{array}{r}53212 \\
3355\end{array}\end{array}$} \\
\hline $12^{\circ} \mathrm{C}$ & $\begin{array}{l}\overline{\mathbf{x}} \\
\pm \text { sd }\end{array}$ & & & & & \\
\hline $18^{\circ} \mathrm{C}$ & $\begin{array}{l}\overline{\mathbf{x}} \\
\pm \mathrm{sd}\end{array}$ & $\begin{array}{r}48862 \\
3033\end{array}$ & $\begin{array}{r}56722 \\
2852\end{array}$ & $\begin{array}{r}65865 \\
1494\end{array}$ & $\begin{array}{r}94104 \\
2669\end{array}$ & $\begin{array}{r}104769 \\
3216\end{array}$ \\
\hline $24^{\circ} \mathrm{C}$ & $\begin{array}{l}\bar{x} \\
\pm \text { sd }\end{array}$ & $\begin{array}{r}50064 \\
1419\end{array}$ & $\begin{array}{r}80780 \\
5865\end{array}$ & $\begin{array}{r}84536 \\
1860\end{array}$ & $\begin{array}{r}79466 \\
1548\end{array}$ & $\begin{array}{r}96190 \\
3200\end{array}$ \\
\hline
\end{tabular}

\section{Energy budget}

The data for the growth rate $(G)$ are calculated from the regression constants for the individual energy content $\left(\mathrm{mJ} \times \mathrm{Ind}^{-1}\right.$, Table 2), which are based on the elemental composition of the larval stages of Elminius modestus. The time spans for the development of the single larval stages are given by Harms (1984). The growth rate of each nauplius stage was calculated therefore by the difference of the energy content at the beginning and at the end of each larval stage. The energy contents of the exuviae (Ex) are presented in Table 3. The metabolism (M) was calculated from the respiration rate given in Figure 1. The values for the food uptake (I) of the naupliar stages are given in Figure 4. The egestion and excretion values (E) were calculated by the difference between the assimilated energy (A) and the ingested food (I) (see "Materials and Methods").

Table 7 shows the energy equations for the single naupliar stages per hour as well as for the development of each nauplius stage and for the total development from nauplius II to the cypris stage. The growth rate of the larvae per hour generally increases with temperature and developmental stage. This is also true for the development of the single naupliar stages, except for the nauplius stages III $\left(18\right.$ and $\left.24^{\circ} \mathrm{C}\right), \operatorname{IV}\left(18^{\circ} \mathrm{C}\right)$ and VI $\left(24^{\circ}\right)$, which show a reduced growth rate compared to lower temperatures, mainly caused by the relatively short developmental time of these three stages (Harms, 1984). All naupliar stages invest more energy in growth than in metabolism. The proportion of metabolism in the energy turnover in the nauplius stages II-IV was higher at 18 and $24^{\circ} \mathrm{C}$ than at $12{ }^{\circ} \mathrm{C}$; the nauplius stages $\mathrm{V}$ and VI showed a different trend (Table 8).

Figure 5 gives a model for the energy balance at $12^{\circ} \mathrm{C}$, based on the cumulative energy budget (Table 7). The energy content of the freshly metamorphosed barnacle can be calculated by the energy content of the freshly hatched larvae ( $5.16 \mathrm{~mJ}$; Harms, 1985), which represents the energy reserve of the egg, the increase of the larval energy content during development to the cypris $(G=109.7 \mathrm{~mJ})$ and the energy loss during the lifetime of the cypris larva, which is a non-feeding stage. The cypris loses $6.95 \mathrm{~mJ}$ with the exuviae (Table 7) and, depending on the duration of this larval stage, $3.26 \mathrm{~mJ} \times$ day $^{-1}$ were used for metabolism. Normally, cypris larvae metamorphosed during the first two days, so that an energy loss of $6.52 \mathrm{~mJ}$ for metabolism was assumed. Young barnacles, therefore, have an energy content of about $100 \mathrm{~mJ}\left(12^{\circ} \mathrm{C}\right), 130 \mathrm{~mJ}\left(24^{\circ} \mathrm{C}\right)$ and $150 \mathrm{~mJ}$ $\left(18^{\circ} \mathrm{C}\right)$. The total energy content of the larvae increased during larval development by a factor of 21 at $12^{\circ} \mathrm{C} ; 25$ at $24^{\circ} \mathrm{C}$ and 31 at $18^{\circ} \mathrm{C}$. 





Table 8. Percentage loss of assimilated energy $(W+E x+M)$ by metabolism at each nauplius stage of Elminius modestus

\begin{tabular}{|cccccc|}
\hline Nauplius stage & II & III & IV & V & VI \\
\hline $12^{\circ} \mathrm{C}$ & 23.9 & 29.3 & 31.3 & 28.2 & 18.6 \\
$18^{\circ} \mathrm{C}$ & 30.7 & 33.2 & 34.9 & 27.3 & 14.6 \\
$24^{\circ} \mathrm{C}$ & 26.1 & 30.3 & 31.7 & 27.4 & 16.8 \\
\hline
\end{tabular}


$E=382.03 \mathrm{~m}$
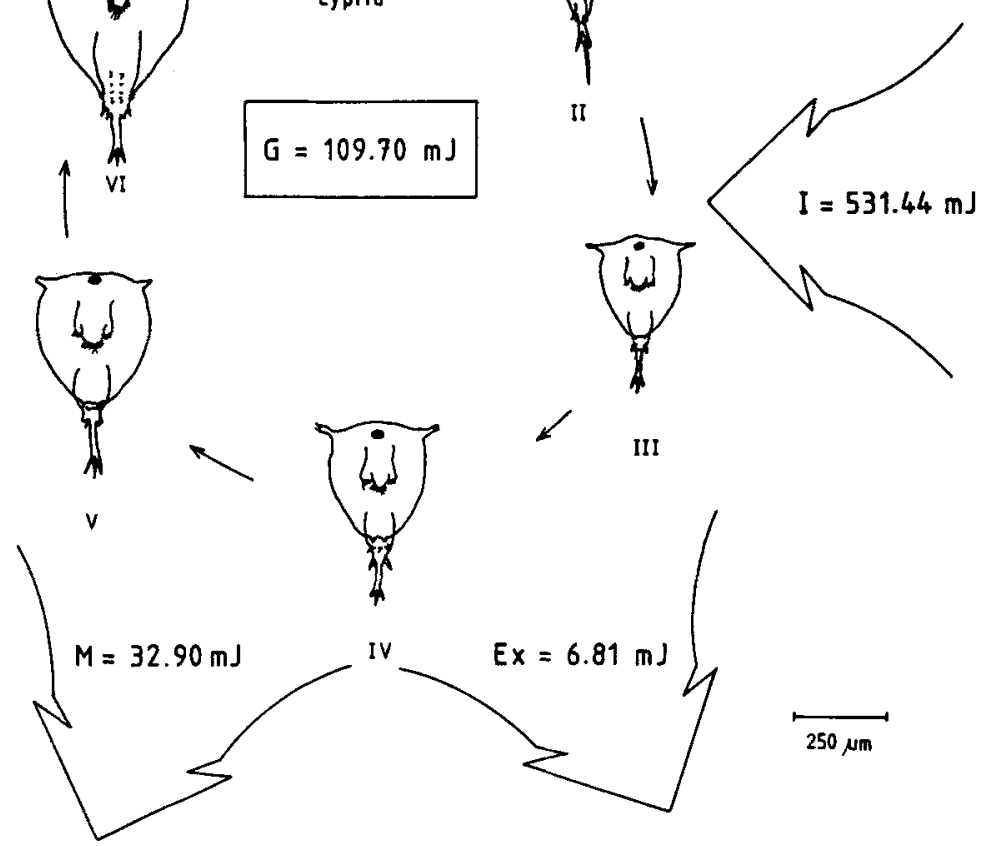

III 

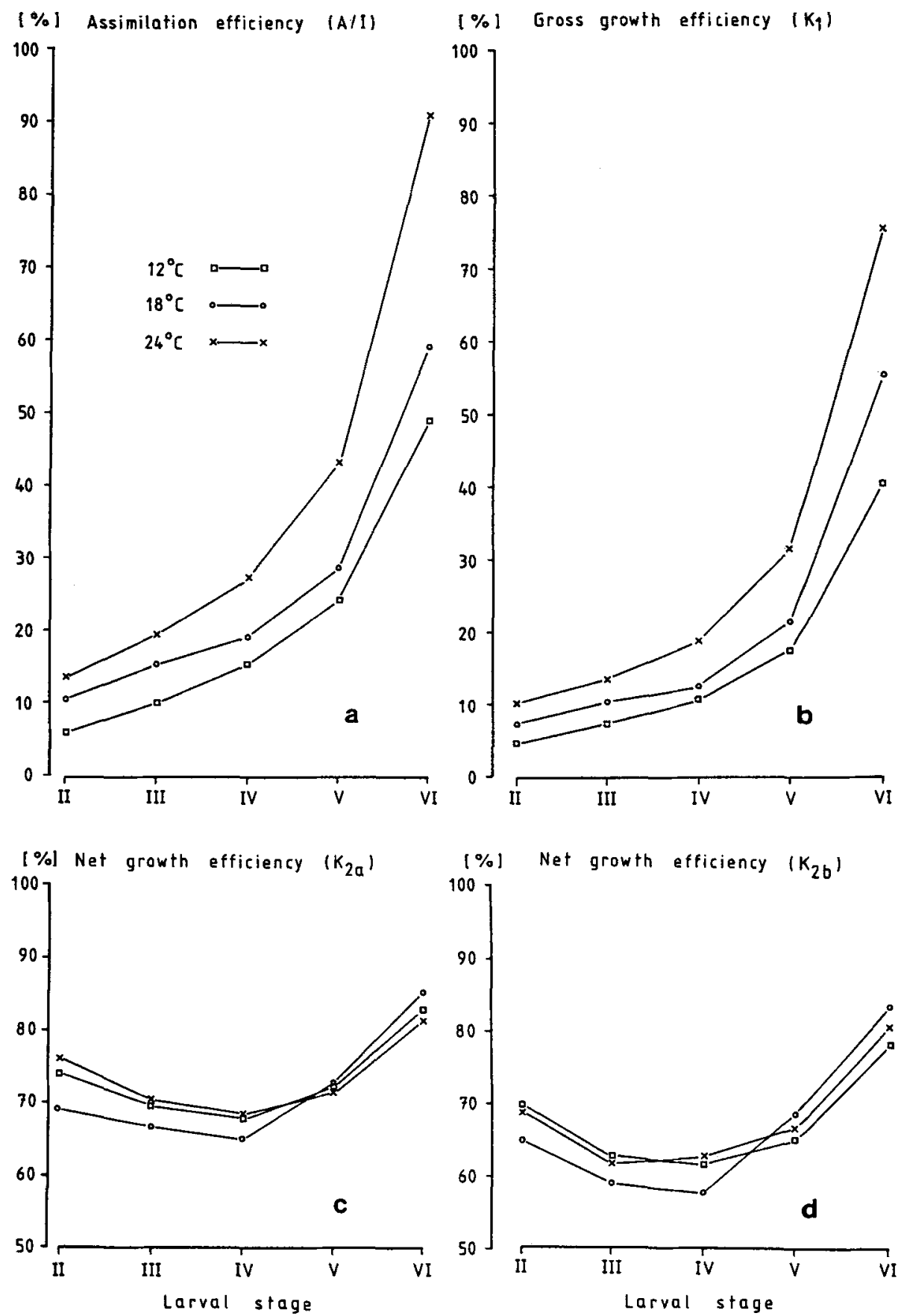

Fig. 6. Efficiences for the naupliar stages II to VI of Elminius modestus. Food concentration: 100 cells Skeletonema costatum $\times \mu \mathrm{l}^{-1}$ 


\section{Efficiencies}

The assimilation efficiency increased exponentially from $6-14 \%$ in nauplius II to $50-90 \%$ in nauplius VI. Highest efficiencies were found at $24{ }^{\circ} \mathrm{C}$ (Fig. 6).

The gross growth efficiency increased exponentially from 4 to $75 \%$ (Fig. 6), showing a similar relationship to temperature and developmental stage as the assimilation efficiency. The net growth efficiency $\left(\mathrm{K}_{2 \mathrm{a}}\right.$ and $\mathrm{K}_{2 \mathrm{~b}}$ ) ranged from 57 to $83 \%$ and showed therefore a much more constant behaviour than the assimilation- and gross growth efficiencies. The net growth efficiency generally decreased from the second to the fourth nauplius stage and increased again in stage $\mathrm{V}$ and VI; no clear temperature influence was obvious.

The assimilation (A/I) and the gross growth efficiency $\left(\mathrm{K}_{1}\right)$ for the total larval development from nauplius II to the cypris increased with the temperature (Fig. 7). However, the net growth efficiencies $\mathrm{K}_{2 \mathrm{a}}$ and $\mathrm{K}_{2 \mathrm{~b}}$ were nearly constant $\left(\mathrm{K}_{2 \mathrm{a}}: 77-80 \%\right.$; $\mathrm{K}_{2 \mathrm{~b}}: 73-77 \%$ ) at the three temperatures tested, with a slight maximum at $18^{\circ} \mathrm{C}$.

\section{DISCUSSION}

\section{Elemental composition}

The dry weight of the second nauplius stage of Elminius modestus given by Bhatnagar \& Crisp (1965) (Table 9) agrees well with the one given in Table 1. The mean dry weight of the larvae of $E$. modestus increased exponentially with the duration of the experiment (Table 2). The specific growth rate of the larval development of $E$. modestus was $0.2-0.5$, depending on the temperature. There are no comparable data of other cirripedes, but these are high values compared with the growth of other crustaceans, whose larvae also show an exponential growth (Dawirs, 1982; Johns \& Pechenik, 1980; Logan \& Epifanio, 1978; Mootz \& Epifanio, 1974).

The percentage content of carbon, nitrogen and hydrogen, as well as the $\mathrm{C}: \mathrm{N}$ ratio of the larvae of $E$. modestus show good agreement with other zooplankton organisms (Beers, 1966; Childress \& Nygaard, 1974; Clutter \& Theilaker, 1971; Ikeda, 1974; Mayzaud, 1973, 1976; Omori, 1969).

\section{Respiration}

The respiration values given by Bhatnager \& Crisp (1965) (Table 9) show good agreement with those given in Table 5. The values of Lucas et al. (1979) for Semibalanus balanoides, and of Jörgensen \& Vernberg (1982) for Balanus eburneus are best compared by the weight specific respiration rate (Tables 9 and 5). B. eburneus, a subtropical species, has higher weight specific respiration rates than Elminius modestus at $24^{\circ} \mathrm{C}$. The native barnacle $S$. balanoides showed only slightly reduced weight specific respiration rates $(1.0-1.9$; Lucas, 1979$)$ when compared with the theoretical values for $E$. modestus at $10^{\circ} \mathrm{C}(1.8-2.7)$.

The respiration rates of the larvae of $E$. modestus are strongly influenced by water temperature. The temperature influence between $12-18^{\circ} \mathrm{C}$ was higher than between $18-24{ }^{\circ} \mathrm{C}$ (Table 10). Such a relative temperature-independent range (low $Q_{10^{-}}$-values between 18 and $24^{\circ} \mathrm{C}$ ) is often found at the temperature range to which the animals are 


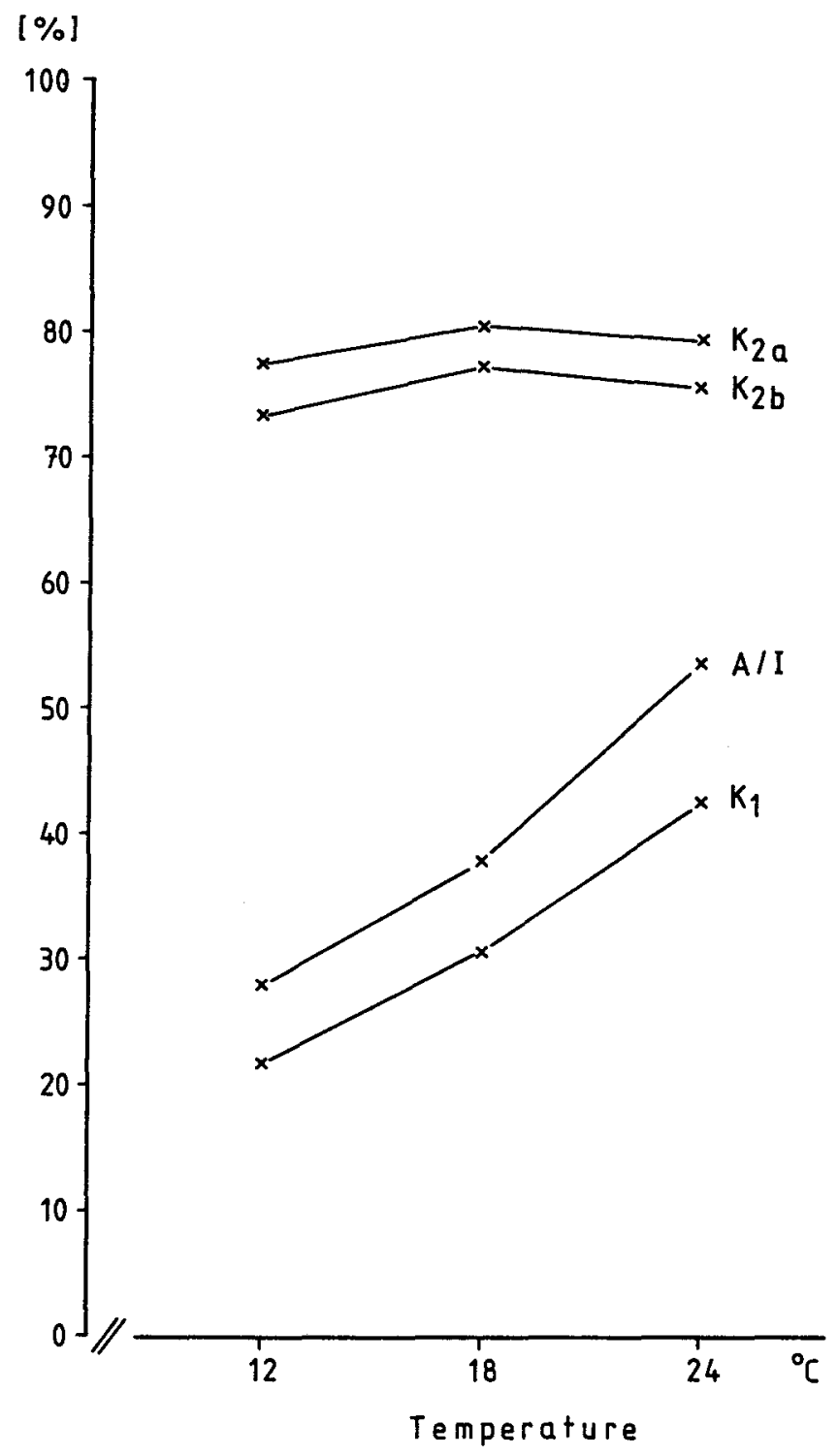

Fig. 7. Efficiences for the total larval development of Elminius modestus from nauplius II to the cypris stage at 12,18 and $24^{\circ} \mathrm{C}$ (Food concentration: 100 cells Skeletonema costatum $\times \mu l^{-1}$ )

adapted (Wieser, 1973). This can be correlated with the original subtropical habitat of the species studied (Foster, 1978; Luckens, 1976).

The cypris larvae consume less oxygen than the last nauplius stage. Similar results were found by Jörgensen \& Vernberg (1982). Lucas et al. (1979) described a further decrease of oxygen uptake when the cypris larvae of $S$. balanoides start to explore the substrate. Since cypris larvae do not feed, they have to use their energy reserves economically. 
Table 9. Summary of literature values for the dry weight and respiration rate of Cirrepedia larvae

\begin{tabular}{|c|c|c|c|c|c|c|}
\hline Species & $\begin{array}{l}\text { Larval } \\
\text { stage }\end{array}$ & $\begin{array}{c}\text { Dry } \\
\text { weight } \\
(\mu g)\end{array}$ & $\begin{array}{l}\text { Respira } \\
\text { ng O} \\
\text { Xind.-1 }_{\mathrm{h}^{-1}}\end{array}$ & $\begin{array}{l}\text { tion rate } \\
\qquad \begin{array}{c}\mathrm{ng} \mathrm{O} \mathrm{O}_{2} \\
\times \mu \mathrm{g} \\
\mathrm{W}^{-1} \times \mathrm{h}^{-1}\end{array}\end{array}$ & $\begin{array}{l}\text { Tempe- } \\
\text { rature } \\
\left({ }^{\circ} \mathrm{C}\right)\end{array}$ & Author \\
\hline E. modestus & II & 0.3 & $2.1-3.1$ & $7.0-10.3$ & 16 & Bhatnager \& Crisp (1965) \\
\hline S. balanoides & $\begin{array}{c}\text { I } \\
\text { II } \\
\text { IV } \\
\text { IV } \\
\text { Cypris } \\
\text { Cypris }\end{array}$ & $\begin{array}{r}0.63 \\
\\
9.8 \\
5.1 \\
33.2 \\
32.0\end{array}$ & $\begin{array}{r}4.7 \\
5.1 \\
14.0 \\
14.0 \\
52.2 \\
30.0\end{array}$ & $\begin{array}{l}5.2 \\
\\
1.0 \\
1.9 \\
1.1 \\
0.6\end{array}$ & $\begin{array}{l}10 \\
10 \\
10 \\
10 \\
10 \\
10\end{array}$ & $\begin{array}{l}\text { Lucas (1979) } \\
\text { Lucas }(1979) \\
\text { Lucas }(1979) \\
\text { Lucas }(1979) \\
\text { Lucas }(1979) \\
\text { Lucas }(1979)\end{array}$ \\
\hline S. balanoides & $\begin{array}{l}\text { Cypris } \\
\text { (swimming) } \\
\text { Cypris } \\
\text { (substrate } \\
\text { exploring) }\end{array}$ & & $\begin{array}{l}52.8 \\
30.0\end{array}$ & & $\begin{array}{l}10 \\
10\end{array}$ & $\begin{array}{l}\text { Lucas et al (1979) } \\
\text { Lucas et al (1979) }\end{array}$ \\
\hline S. balanoides & Cypris & 37.7 & & & & Holland \& Walker (1975) \\
\hline B. eburneus & $\begin{array}{l}\text { I } \\
\text { IV } \\
\text { VI } \\
\text { Cypris }\end{array}$ & $\begin{array}{l}0.27 \\
0.68 \\
1.5 \\
2.18\end{array}$ & $\begin{array}{l}6.97 \\
23.6 \\
71.7 \\
13.4\end{array}$ & $\begin{array}{l}25.8 \\
34.7 \\
47.8 \\
6.15\end{array}$ & $\begin{array}{l}24 \\
24 \\
24 \\
24\end{array}$ & $\begin{array}{l}\text { Jorgensen \& Vernberg (1982) } \\
\text { Jorgensen \& Vernberg (1982) } \\
\text { Jorgensen \& Vernberg (1982) } \\
\text { Jorgensen \& Vernberg (1982) }\end{array}$ \\
\hline
\end{tabular}

Table $10 . Q_{10}$-values for the respiration rate at different temperature intervals

\begin{tabular}{|cccccccc|}
\hline $\begin{array}{c}\text { Larval } \\
\text { stage }\end{array}$ & $\begin{array}{c}\text { II } \\
\text { (newly } \\
\text { hatched) }\end{array}$ & II & III & IV & V & VI & Cypris \\
\hline $12-18^{\circ} \mathrm{C}$ & 2.70 & 4.50 & 3.62 & 4.95 & 2.82 & 2.33 & 1.91 \\
$18-24^{\circ} \mathrm{C}$ & 2.45 & 1.41 & 1.94 & 1.71 & 2.29 & 2.01 & 1.73 \\
$12-24^{\circ} \mathrm{C}$ & 2.58 & 2.52 & 2.65 & 2.91 & 2.54 & 2.16 & 1.82 \\
\hline
\end{tabular}

\section{Food uptake}

There are no directly comparable data for the food uptake of other cirripede larvae. Walne $(1965,1966)$ found an ingestion rate of 5.5-6.0 $\times 10^{4}$ cells $\times$ day $^{-1}$ for young Ostrea edulis (shell length $180-260 \mu \mathrm{m}$ ). Similar ingestion rates were reported by Malouf \& Breese (1977) for Crassostrea edulis (shell length $>200 \mu \mathrm{m}$ ) with $6.2 \times 10^{4}$ cells $\times$ day $^{-1}$. Sprung (1984) gave a general review on the ingestion rates of bivalves.

The maximum food uptake of copepods was estimated at $29 \times 10^{4}$ cells $\times$ day (Frost, 1972) and the mean ingestion rate was $11 \times 10^{4}$ cells $\times$ day $^{-1}$. The food uptake of Elminius modestus (Table 6) showed a similar magnitude and varied between 4.1-10.5 $\times 10^{4}$ cells $\times$ days $^{-1}$, depending on temperature and developmental stage. 


\section{Energy budget}

The energy loss by metabolism during the development of the naupliar stages of Elminius modestus was low compared with the energy invested in growth. The part of the assimilated energy $(W+E x+M)$ which was used up by metabolism increased from the second to the fourth nauplius stage to a maximum of $31-34 \%$. This percentage decreased in the last nauplius stage to $14-19 \%$ (Table 8 ). The proportion of the metabolism showed a similar behaviour at the three temperatures tested, and was highest at $18^{\circ} \mathrm{C}$ (stage II-IV).

The energy utilization for metabolism during the larval development of decapods often shows a much higher percentage during early stages (Dawirs, 1982; Logan \& Epifanio, 1978; Mootz \& Epifanio, 1974), whereas the metabolism proportion is often reduced in older larval stages. Levine \& Sulkin (1979) reported that larvae of $R$ hithropanopeus harrisii also invest more energy in growth than in metabolism.

The energy loss by the exuviae lies between 2-8\% of the assimilated energy in each nauplius stage and reached its minimum at stage VI (Table 11). The energy loss by the exuviae on the growth rate during each single nauplius stage was $2-12 \%$ and the lowest value was again found in the last nauplius stage (Table 4, numbers in brackets). Lei \& Armitage (1980) found similar percentages in energy loss by the exuviae of Daphnia ambigua. Branchinecta gigas lose $5-6 \%$ of the assimilated energy with the exuviae (Daborn, 1975). Young isopods of Idothea baltica and Sphaermonas pulchellum lose $7-13 \%$ of the assimilated energy with the exuviae (Tsikhon-Iukania \& Lukasheva, 1970 ). Adult individuals often lose higher percentages of energy with their exuviae (Lei \& Armitage, 1980).

Table 11. Percentage energy loss of assimilated energy $(W+E x+M)$ by the exuviae of each nauplius stage of Elminius modestus in mJoule

\begin{tabular}{|cccccc|}
\hline Nauplius stage & II & III & IV & V & VI \\
\hline $12^{\circ} \mathrm{C}$ & 6.6 & 8.0 & 7.3 & 6.7 & 3.3 \\
$18^{\circ} \mathrm{C}$ & 4.9 & 8.0 & 7.6 & 4.1 & 2.2 \\
$24^{\circ} \mathrm{C}$ & 5.0 & 8.3 & 5.6 & 5.6 & 2.5 \\
\hline
\end{tabular}

\section{Efficiencies}

Assimilation and gross growth efficiencies describe the food utilization under special conditions; consequently, they are not constants. Food concentration and food value strongly influence the assimilation efficiency, because different compounds can be assimilated at different proportions (Corner \& Davies, 1971).

Assimilation efficiency (A/I): The assimilation efficiency of aquatic crustaceans varies between 7-99\% (Shuschenya, 1969). Conover (1964) and Corner \& Cowey (1968) found values of 7-99\% for zooplankton.

The assimilation efficiency of the larval stages of Elminius modestus increased from 6.3-91.2\%. Lucas (1979) reported a similar increase for the larval stages of Semibalanus balanoides $(5.0-42 \%)$. The reason for such an increase of the assimilation efficiency during the larval development might be due to a reduction of the relative respiration rate 
(Fig. 3) or a better efficiency of enzymes (Needham, 1931: in Calow, 1977b). Another explanation for the increase of the assimilation efficiency might also be found in the food uptake of the larvae. The young larval stages of $E$. modestus have to break the chains of Skeletonema costatum before ingestion whereas the older stages can ingest these chains more easily.

Gross growth efficiency $\left(K_{1}\right)$ : Blaxter (1962: in Calow, 1977b) found that $K_{1}$-values rarely are $>35 \%$. These results are based on homoiotherms. Poikilotherms can reach higher values, because they do not have to utilize energy for temperature regulation (Calow, 1977a). Calow (1977b) therefore found $\mathrm{K}_{1}$-values up to $50 \%$ for poikilotherms. This is in good agreement with the gross efficiency of the larval development of $E$. modestus which increased from 4 to $50 \%\left(12{ }^{\circ} \mathrm{C}\right.$ and $\left.18{ }^{\circ} \mathrm{C}\right)$ and reached higher values $(75.4 \%)$ only at the sixth nauplius stage at $24^{\circ} \mathrm{C}$.

Net growth efficiency $\left(K_{2}\right)$ : The net growth efficiencies were relatively constant throughout the larval development of $E$. modestus. Lucas (1979) found net growth efficiencies of $61-83 \%$ for larvae of $S$. balanoides at $10^{\circ} \mathrm{C}$, which is expected to be the optimum temperature for the larval development of $S$. balanoides. These values are very similar to the one found for $E$. modestus at the temperature interval tested (Fig. 6). Generally, herbivores have lower assimilation- and higher net growth efficiencies than carnivores (Welch, 1968). In contrast to the assimilation and gross growth efficiency, the net growth effficiency for the total larval development of $E$. modestus showed only a slight maximum at $18^{\circ} \mathrm{C}$ (Fig. 7) and was scarcely influenced by temperature. This high net growth efficiency over the tested temperature interval $\left(12^{\circ} \mathrm{C}-24^{\circ} \mathrm{C}\right)$ was certainly one of the preadaptations for the successful immigration of $E$. modestus throughout Europe.

Acknowledgements. This work was supported by a grant of the "Studienstiftung des deutschen Volkes". I am grateful to Dr. A. Buschinger (TH Darmstadt) who supervised this work and to Dr. O. Kinne and Dr. H.-P. Bulnheim who provided working facilities at the Marine Biological Station Helgoland (BAH). My thanks are also due to all members of the BAH who supported this work: Dr. E. Hagmeier helped in cultivating food organisms (Skeletonema costatum); the elemental analyses were carried out by Ms. C. Püschel; B. Seeger typed the manuscript, and Dr. M. Rieper corrected the English manuscript. In particular I am thankful to Dr. K. Anger (BAH) for his helpful comments and fruitful discussions. This paper is part of a dissertation (TH Darmstadt).

\section{LITERATURE CITED}

Anger, K. \& Jacobi, C. C., 1985. Respiration and growth of Hyas araneus L. larvae (Decapoda, Majidae) from hatching to metamorphosis. - J. exp. mar. Biol. Ecol. 88, 257-270.

Barnes, H. \& Barnes, M., 1958. The rate of development of Balanus balanoides (L.) larvae. - Limnol. Oceanogr. 3, 29-32.

Beers, J. R., 1966. Studies on the chemical composition of the major zooplankton groups in the Sargasso Sea off Bermuda. - Limnol. Oceanogr. 11, 520-528.

Belman, B. W. \& Childress, J. J., 1973. Oxygen consumption of the larvae of the lobster Panulirus interruptus (Randall) and the crab Cancer productus Randall. - Comp. Biochem. Physiol, 44A, 821-828.

Bhatnager, K. M. \& Crisp, D. G., 1965. The salinity tolerance of nauplius larvae of cirripedes. - J. Anim. Ecol, 34, 419-428.

Bishop, M. W. H., 1947. Establishment of an immigrant in British coastal waters. - Nature, Lond. 159, 501. 
Calow, P., 1977a. Ecology, evolution and energetics: A study in metabolism adaptation. - Adv. ecol. Res. 10, 1-10.

Calow, P., 1977b. Conversion efficiencies in heterotrophic organisms. - Biol. Rev. 52, 385-409.

Childress, J. J. \& Nygaard, M., 1974. Chemical composition and buoyancy of midwater crustaceans as function of depth of occurrence off southern California. - Mar. Biol. 27, 225-238.

Clutter, R. I. \& Theilacker, G. H., 1971. Ecological efficiency of a pelagic mysid shrimp: Estimates from growth, energy budget, mortality studies. - Fish. Bull. U. S. 69, 93-115.

Conover, R. J., 1964. Food relations and nutrition of zooplankton. - Occ. Publs Narragansett mar. Lab. 2, 81-89.

Corner, E. D. S. \& Cowey, C. B., 1968. Biochemical studies on the production of marine zooplankton. - Biol. Rev. 43, 393-426.

Corner, E. D. S. \& Davies, A. G., 1971. Plankton as a factor in the nitrogen and phosphorus cycles in the sea. - Adv. mar. Biol. 9, 101-204.

Crisp, D. J., 1974. Energy relations of marine invertebrate larvae. - Thalassia jugosl. 10, $103-120$.

Crisp, D. J., 1976. The role of the pelagic larvae. In: Perspectives in experimental biology. Ed. by P. Spencer-Davies. Pergamon Press, Oxford 1, 145-155.

Crisp, D. J., 1984. Energy flow measurements. In: Methods for the study of marine benthos. Ed. by N. A. Holme \& A. D. McIntyre. Blackwell, Oxford, 284-365.

Daborn, G. R., 1975. Life history and ecology relations of the giant fairy shrimp Branchinecta gigas Lynch 1937 (Crustacea, Anostraca). - Ecology 56, 1023-1039.

Dawirs, R. R., 1982. Laboruntersuchungen zur larvalen Entwicklung von Carcinus maenas L. (Decapoda, Portunidae). Diss., Univ. Kiel, 189 pp.

Dawirs, R. R., 1983. Respiration, energy balance and developmental pattern in growing and starving larvae of Carcinus maenas L. (Decapoda, Portunidae). - J. exp. mar. Biol. Ecol. 69, 105-128.

Finlay, B. J. \& Uhlig, G., 1981. Calorific and carbon values of marine and freshwater protozoa. Helgoländer wiss. Meeresunters. 34, 401-412.

Foster, B. A., 1978. The marine fauna of New Zealand: Barnacles (Cirripedia: Thoracica). - Mem. N. Z. oceanogr. Inst. $69,1-160$.

Frost, B. W., 1972. Effects of size and concentration of food particles on the feeding behavior of the marine planktonic copepod Calanus pacificus. - Limnol. Oceanogr. 17, 805-815.

Gnaiger, E., 1983. Calculation on energètic and biochemical equivalents of respiratory oxygen consumption. In: Polarographic oxygen sensors. Ed. by E. Gnaiger \& H. Forstner. Springer, Berlin, 337-345.

Grasshoff, K., 1976. Methods of sea water analysis. Verl. Chemie, Weinheim, 317 pp.

Guillard, R. R. L., 1978. Counting slides. In: Phytoplankton manual. Ed. by A. Sournia. Unesco, Paris, 182-189.

Harms, J., 1984. Influence of water temperature on larval development of Elminius modestus and Semibalanus balanoides (Crustacea, Cirripedia). - Helgoländer Meeresunters. 38, 123-134.

Harms, J., 1985. Untersuchungen zum Lebenszyklus von Elminius modestus Darwin (Crustacea, Balanoidea). Diss., TH Darmstadt, 146 pp.

Harms, J., 1986. Effects of temperature and salinity on larval development of Elminius modestus (Crustacea, Cirripedia) from Helgoland (North Sea) and New Zealand. - Helgoländer Meeresunters. 40, 355-376.

Holland, D. L. \& Walker, G., 1975. The biochemical composition of the cypris larva of the barnacle Balanus balanoides L. - J. Cons. int. Explor. Mer 36, 162-165.

Ikeda, T., 1974. Nutritional ecology of marine zooplankton. - Mem. Soc. Fish. Hokkaido Univ. 22, 1-97.

Jörgensen, D. D. \& Vernberg, W. B., 1982. Oxygen uptake in a barnacle: Scaling to body size from nauplius to adult. - Can. J. Zool. 60, 1231-1235.

Johns, D. M. \& Pechenik, J. A., 1980. Influence of the water-accommodated fraction of Nr. 2 fuel oil on energetics of Cancer irroratus larvae. - Mar. Biol. 35, 247-254.

Lei, Chi-Hisang \& Armitage, K. B., 1980. Energy budget of Daphnia ambigua Scourfield. - J. Plankt. Res. 2, 261-281.

Levine, D. M. \& Sulkin, S. D., 1979. Partitioning and utilization of energy during larval development of the xanthid crab Rhithropanopeus harrisii (Gould). - J. exp. mar. Biol. Ecol. 40, 247-257. 
Logan, D. T. \& Epifanio, C. E., 1978. A laboratory energy balance for the larvae and juveniles of the American lobster Homarus americanus. - Mar. Biol. 47, 381-389.

Lucas, M. I., 1979. Studies on energy flow in a barnacle population. Thesis, Univ. of Wales, $257 \mathrm{pp}$.

Lucas, M. I., Walker, G., Holland, D. L. \& Crisp, D. J., 1979. An energy budget for the free-swimming and metamorphosing larvae of Balanus balanoides (Crustacea; Cirripedia). - Mar. Biol. 55, 221-229.

Luckens, P. A., 1976. Settlement and succession on rocky shores at Auckland, North Island, New Zealand. - Mem. N. Z. oceanogr. Inst. 70, 1-64.

Malouf, R. E. \& Breese, W. P., 1977. Food consumption and growth of larvae of the pacific oyster, Crassostera gigas (Thunberg), in a constant flow rearing system. - Proc. natn. Shellfish. Ass. 67, 7-16.

Mayzaud, P., 1973. Respiration and nitrogen excretion of zooplankton. II. Studies of the metabolic characteristics of starved animals. - Mar. Biol. 21, 19-28.

Mayzaud, P., 1976. Respiration and nitrogen excretion of zooplankton. IV. The influence of starvation on the metabolism and the biochemical composition of some species. - Mar. Biol. 37, 47-58.

Molenock, J. \& Gomez, E. D., 1972. Larval stages and settlement of the barnacle Balanus (conopea) galeatus (L.) (Cirripedia, Thoracica). - Crustaceana 23, 100-108.

Mootz, C. A. \& Epifanio, C. E., 1974. An energy budget for Menippe mercenaria larvae fed Artemia nauplii. - Biol. Bull. mar. biol. Lab., Woods Hole, 146, 44-45.

Moyse, J., 1963. A comparison of the value of various flagellates and diatoms as food for barnacle larvae. - J. Cons. int. Explor. Mer 28, 175-187.

Omori, M., 1969. Weight and chemical composition of some important oceanic zooplankton in the North Pacific Ocean. - Mar. Biol. 3, 4-10.

Salonen, K., Sarvala, J., Hakula, I. \& Viljanen, M.-L., 1976. The relation of energy and organic carbon in aquatic invertebrates. - Limnol. Oceanogr. 21, 724-730.

Shuschenya, L. M., 1969. Quantitative relations of metabolism and transformation of matter and energy in Crustacea. - Transl. Ser. Fish. Res. Bd Can. 1374.

Sprung, M., 1984. Physiological energetics of mussel larvae (Mytilus edulis). II. Food uptake. - Mar. Ecol. Prog. Ser. 17, 295-305.

Strathmann, R. R., 1967. Estimating the organic carbon of phytoplankton from cell volume or plasma volume. - Limnol. Oceanogr. 12, 411-418.

Tighe-Ford, D. J., Power, M. J. D. \& Vaile, D. C., 1970. Laboratory rearing of barnacles larvae for antifouling research. - Helgoländer wiss. Meeresunters. 20, 393-405.

Tsikhon-Lukania, Y. A. \& Lukasheva, T. A., 1970. Conversion of food energy in the young of some marine isopods. - Oceanology 10, 553-556.

Utermöhl, H., 1958. Zur Vervollkommnung der quantitativen Phytoplankton Methodik. - Mitt. int. Ver. theor. angew. Limnol. 9, 1-38.

Walley, L. J., 1969. Studies on the larval structure and metamorphosis of Balanus balanoides. - Phil. Trans. R. Soc. (B) 256, 237-280.

Walne, P. R., 1965. Observations on the influence of food supply and temperature on the feeding and growth of the larvae of Ostrea edulis. - Fishery Invest., Lond. (Ser. 2) 24, 1-45.

Walne, P. R., 1966. Experiments in the large scale culture of the larvae of Ostrea edulis L. - Fishery Invest., Lond. (Ser. 2) 25, 1-53.

Welch, H. E., 1968. Relationships between assimilation efficiencies and growth efficiencies for aquatic consumers. - Ecology 49, 755-759.

Wieser, W., 1973. Temperature relations of ectotherms: A speculative review: In: Effects of temperature on ectothermic organisms. Ed. by W. Wieser. Springer, Berlin, 1-23.

Wisely, B., 1960. Experiments on rearing the barnacle Elminius modestus Darwin to the settling stage in the laboratory. - Aust. J. mar. Freshwat. Res. 11, 42-54. 